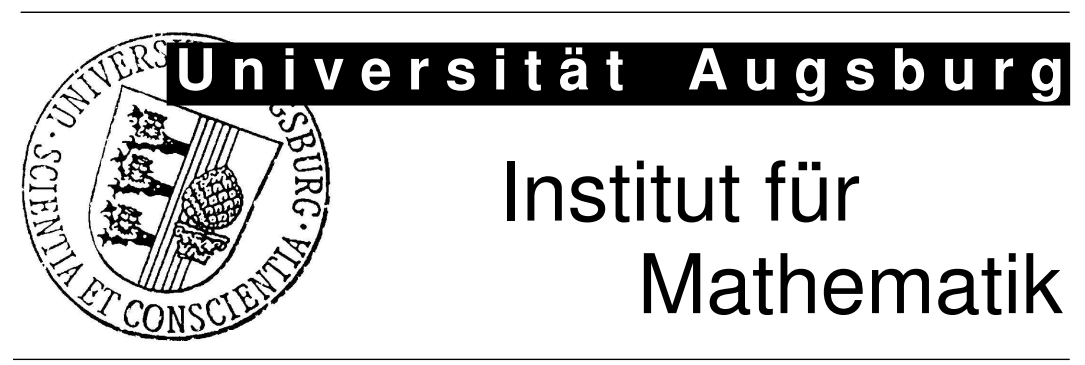

Martin Rasmussen

Bifurcations of Asymptotically Autonomous Differential Equations

Preprint Nr. 10/2008 - 25. Februar 2008

Institut für Mathematik, Universitätsstraße, D-86135 Augsburg

http: //www.math. uni-augsburg.de/ 


\section{Impressum:}

Herausgeber:

Institut für Mathematik

Universität Augsburg

86135 Augsburg

http://www . math. uni-augsburg.de/forschung/preprint/

ViSdP:

Martin Rasmussen

Institut für Mathematik

Universität Augsburg

86135 Augsburg

Preprint: Sämtliche Rechte verbleiben den Autoren (C) 2008 


\title{
BIFURCATIONS OF ASYMPTOTICALLY AUTONOMOUS DIFFERENTIAL EQUATIONS
}

\author{
MARTIN RASMUSSEN
}

\begin{abstract}
This article deals with bifurcation phenomena of asymptotically autonomous differential equations. Under the assumption that the underlying autonomous system admits a bifurcation of pitchfork, saddle node, transcritical or Hopf type, nonautonomous bifurcation results are obtained for both the bifurcation of attraction and repulsion areas and transitions of attractors and repellers.
\end{abstract}

\section{INTRODUCTION}

The theory of nonautonomous bifurcation phenomena is currently a field of active research (see the recent papers $[8,11,13,14,15,16,17,19,22,23,24]$ ), but hitherto, there is no established notion of a nonautonomous bifurcation. When developing general notions for nonautonomous systems, e.g., notions of bifurcation, one should also pay attention to autonomous systems in the sense that the new concepts should fully reflect the autonomous situation. Moreover, both for testing the notions and elucidation, it is reasonable to consider asymptotically autonomous systems in a next step. In fact, useful nonautonomous concepts of bifurcation should lead to results in the asymptotically autonomous case whenever the underlying autonomous system admits a bifurcation.

This article aims at promoting this paradigm by discussing two different concepts of a nonautonomous bifurcation in the context of asymptotically autonomous differential equations. The study of asymptotically autonomous differential equations goes back to Markus [20], where properties of nonautonomous $\omega$-limit sets are discussed and the Theorem of Poincaré \& Bendixson is generalized to asymptotically autonomous planar systems. It is not clear a priori, however, if certain behavior carries over from the autonomous to the nonautonomous system. In fact, in [29], several examples of asymptotically autonomous systems are studied that behave quite differently from the limiting equations. In [18], however, it is shown that the pullback and forward behavior of a special asymptotically autonomous Lotka-Volterra system is consistent to the underlying autonomous system.

The nonautonomous bifurcation concepts used in this paper have been developed in $[26,27,25])$. For a motivation of the new notions, let us first look at a special situation of an autonomous bifurcation. Consider the ordinary differential equation $\dot{x}=x\left(\alpha+x^{2}\right)$ for a real parameter $\alpha$, which is a prototype of a pitchfork bifurcation

2000 Mathematics Subject Classification. 34D05, 34D45, 37B55, 37C70, 37C75, 37G10, $37 \mathrm{G} 35$.

Key words and phrases. Asymptotically autonomous differential equation, attractor, bifurcation, repeller, transition.

Research supported by the Bayerisches Eliteförderungsgesetz of the State of Bavaria and the Graduiertenkolleg Nichtlineare Probleme in Analysis, Geometrie und Physik (GK 283) financed by the DFG. 
as indicated in Figure 1. For $\alpha \geq 0$, there is only one equilibrium, which is given by zero and which is repulsive. By letting the parameter $\alpha$ pass through zero in negative direction, this equilibrium becomes attractive, and two other repulsive equilibria, given by $\pm \sqrt{-\alpha}$, are bifurcating.

In order to establish a nonautonomous bifurcation theory, consider this scenario in the following way: For $\alpha<0$, the trivial solution is attractive, and the domain of attraction $\mathcal{A}(\alpha)$ is given by the open interval between the two other equilibria. Now, the main point is that this domain of attraction undergoes a qualitative change from a nontrivial to a trivial object in the limit $\alpha \nearrow 0$. Moreover, the closure of $\mathcal{A}(\alpha)$ is also a repeller, and thus, also a repeller changes qualitatively for $\alpha \succ 0$. We call the shrinking of a domain of attraction (repulsion, respectively) a bifurcation, whereas the case of a changing repeller (attractor, respectively) is denoted as a transition.

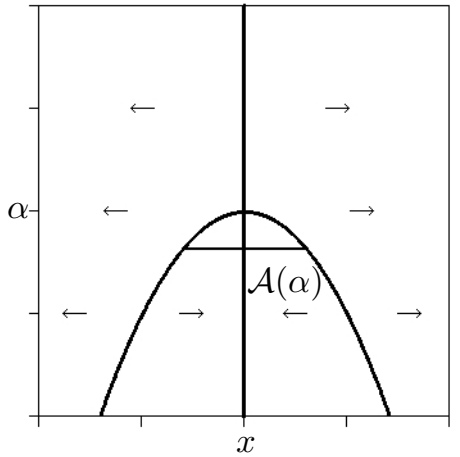

FiguRE 1. Pitchfork bifurcation

To realize this idea in the nonautonomous situation, notions of attractive and repulsive solutions, domains of attractivity and repulsivity, as well as attractor and repeller are needed. It is possible to distinguish between several points of view concerning different time domains. In this article, we will only consider the past of the system, since we are interested in the asymptotic behavior for $t \rightarrow-\infty$. Via time reversal, analogous results are obtained for the future of the system. One can also define concepts which are suitable to capture the dynamical behavior on the whole time axis (see $[25,26,27]$ ).

This paper essentially splits into three parts. The first two sections contain the preparation of basic definitions and notions for general nonautonomous differential equations. Basic results for asymptotically autonomous differential equations are then obtained in Section 4 and Section 5. Finally, the remaining part of the paper concentrates on the study of examples: In Section 6, one-dimensional bifurcations such as the pitchfork, transcritical and saddle node bifurcation are discussed, and Section 7 is devoted to study the Hopf bifurcation scenario.

\section{Preliminaries}

We denote by $\mathbb{R}^{N \times N}$ the set of all real $N \times N$ matrices. The Euclidean space $\mathbb{R}^{N}$ is equipped with the Euclidean norm $\|\cdot\|$, which is induced by the scalar product $\langle\cdot, \cdot\rangle$, defined by $\langle x, y\rangle:=\sum_{i=1}^{N} x_{i}, y_{i}$. We write $U_{\varepsilon}\left(x_{0}\right)=\left\{x \in \mathbb{R}^{N}:\left\|x-x_{0}\right\|<\varepsilon\right\}$ for the $\varepsilon$-neighborhood of some point $x_{0} \in \mathbb{R}^{N}$ and set $U_{\varepsilon}(A):=\cup_{x \in A} U_{\varepsilon}(x)$ for $A \subset \mathbb{R}^{N}$. For arbitrary nonempty sets $A, B \subset \mathbb{R}^{N}$ and $x \in \mathbb{R}^{N}$, let $d(x, A):=$ $\inf \{d(x, y): y \in A\}$ be the distance of $x$ to $A$ and $d(A \mid B):=\sup \{d(x, B): x \in A\}$ be the Hausdorff semi-distance of $A$ and $B$. The closure and the interior of $A$ is denoted by $\operatorname{cls} A$ and $\operatorname{int} A$, and the diameter of $A$ is defined by $\operatorname{diam} A:=$ $\sup _{x, y \in A}\|x-y\|$. Moreover, we set $\mathbb{R}_{\kappa}^{+}:=[\kappa, \infty)$ and $\mathbb{R}_{\kappa}^{-}:=(-\infty, \kappa]$ for $\kappa \in \mathbb{R}$.

Let $g: X \rightarrow Y$ be a function from a set $X$ to a set $Y$. Then the graph of $g$ is defined by graph $g:=\{(x, y) \in X \times Y: y=g(x)\}$. 
Given a differentiable function $g: X \subset \mathbb{R}^{N} \rightarrow \mathbb{R}^{M}$, we write $D g: X \rightarrow \mathbb{R}^{M \times N}$ for its derivative and $D_{i} g: X \rightarrow \mathbb{R}^{M}$ for its partial derivative with respect to the $i$-th variable, $i \in\{1, \ldots, N\}$.

For a continuous function $f: \Omega \subset \mathbb{R} \times \mathbb{R}^{N} \rightarrow \mathbb{R}^{N}$, we use the notation

$$
\dot{x}=f(t, x)
$$

to denote the ordinary differential equation $\dot{x}(t)=f(t, x(t))$. We assume that $f$ fulfills conditions for the local existence and uniqueness of solutions. Let $\lambda$ stand for the general solution of $(2.1)$, i.e., $\lambda(\cdot, \tau) \xi$ is the unique non-continuable solution of (2.1) satisfying the initial condition $\lambda(\tau, \tau) \xi=\xi$. In case (2.1) is autonomous, i.e., independent of $t$, the flow of $(2.1)$ is defined by $\varphi(t, x)=\lambda(t, 0) x$.

For $\tau \in \mathbb{R}$, a subset $M$ of the extended phase space $(-\infty, \tau) \times \mathbb{R}^{N}$ is called a nonautonomous set if for all $t<\tau$, the so-called $t$-fibers $M(t):=\left\{x \in \mathbb{R}^{N}:(t, x) \in\right.$ $M\}$ are nonempty. One could also think of a nonautonomous set as a map from $(-\infty, \tau)$ to the nonempty subsets of $\mathbb{R}^{N}, t \mapsto M(t)$. We call $M$ compact if all $t$-fibers are compact, and $M$ is said to be invariant if $\lambda(t, s) M(s)=M(t)$ for all $t, s<\tau$.

Given a nonautonomous set $M \subset(-\infty, \tau) \times \mathbb{R}^{N}$, we define

$$
\limsup _{t \rightarrow-\infty} M(t):=\bigcap_{\tau \leq 0} \bigcup_{t \leq \tau} M(t) \text { and } \liminf _{t \rightarrow-\infty} M(t):=\bigcup_{\tau \leq 0} \bigcap_{t \leq \tau} M(t)
$$

(see [3]). It is easy to show that

$$
\begin{aligned}
\limsup _{t \rightarrow-\infty} M(t) & =\left\{x \in \mathbb{R}^{N}: \forall \tau \leq 0: \exists t \leq \tau: x \in M(t)\right\} \\
\text { and } \quad \liminf _{t \rightarrow-\infty} M(t) & =\left\{x \in \mathbb{R}^{N}: \exists \tau \leq 0: \forall t \leq \tau: x \in M(t)\right\} .
\end{aligned}
$$

\section{Notions of Attractivity, Repulsivity, Bifurcation and Transition}

In this section, the relevant notions of attractivity and repulsivity to study nonautonomous bifurcation phenomena are introduced (see also [26]). In addition, two examples illustrate the definitions.

The following definition is devoted to the introduction of past attractor and past repeller. Note that a past attractor is a local form of a pullback attractor which is discussed since the 1990s (see, e.g., [6]), i.e., it attracts a neighborhood of itself in the sense of pullback attraction. A past repeller attracts also a neighborhood of itself, but in backward time, i.e., and a past repeller is a local forward attractor for the system under time reversal. See also Figures 2 and 3 for an illustration of these notions.

Definition 3.1 (Past attractivity and repulsivity). Let $\tau$ be real, and let $A, R \subset$ $(-\infty, \tau) \times \mathbb{R}^{N}$ be compact and invariant nonautonomous sets and $\mu:(-\infty, \tau) \rightarrow \mathbb{R}^{N}$ be a solution of (2.1).

(i) $A$ is called a past attractor if there exists an $\eta>0$ with

$$
\lim _{t \rightarrow \infty} d\left(\lambda(s, s-t) U_{\eta}(A(s-t)) \mid A(s)\right)=0 \quad \text { for all } s<\tau .
$$

(ii) $\mu$ is called past attractive if graph $\mu$ is a past attractor.

(iii) $A$ is called a past repeller if there exists an $\eta>0$ with

$$
\lim _{t \rightarrow \infty} d\left(\lambda(s-t, s) U_{\eta}(A(s)) \mid A(s-t)\right)=0 \quad \text { for all } s<\tau .
$$




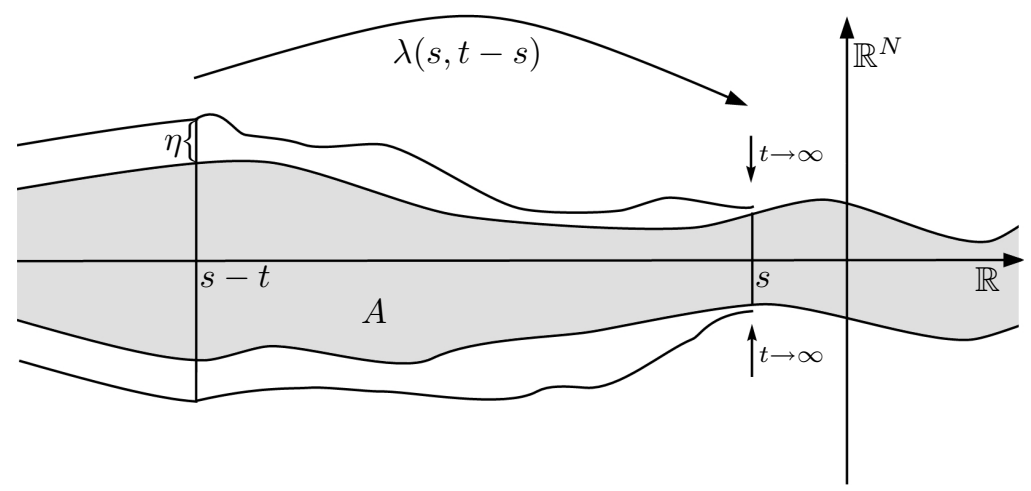

Figure 2. Past attractor $A$

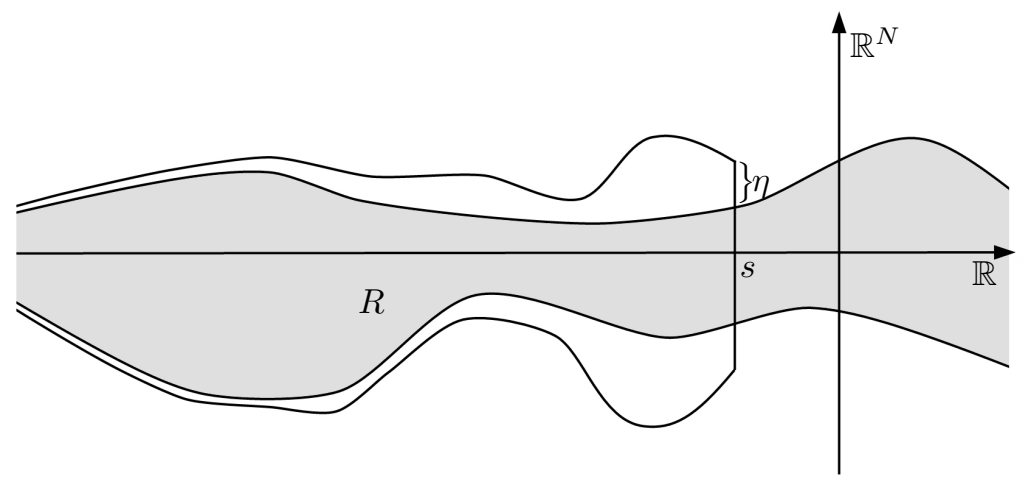

FiguRE 3. Past repeller $R$

(iv) $\mu$ is called past repulsive if graph $\mu$ is a past repeller.

\section{Remark 3.2.}

(i) A past attractor is a local form of a pullback attractor (see, e.g., [6]): Let $\mathcal{D}$ be a collection of nonautonomous sets (often, $\mathcal{D}$ consists of fiberwiseconstant bounded nonautonomous sets). Then a pullback attractor of (2.1) with attraction universe $\mathcal{D}$ is given by a nonempty compact and invariant nonautonomous set $A \subset(-\infty, \tau) \times \mathbb{R}^{N}$ fulfilling

$\lim _{t \rightarrow \infty} d(\lambda(s, s-t) D(s-t) \mid A(s))=0 \quad$ for all $D \in \mathcal{D}$ and $s<\tau$.

Obviously, $A$ is a past attractor if and only if there exists a $\eta>0$ such that with the nonautonomous set $D$, defined by its $t$-fibers $D(t):=U_{\eta}(A(t)), A$ is pullback attractor with attraction universe $\{D\}$. In addition, we refer to [5] for a discussion of several notions of attractivity for nonautonomous differential equations.

(ii) Due to the continuity of the general solution, one can derive the following equivalent characterization: A compact and invariant nonautonomous set 
$A$ is a past attractor if and only if there exists an $\eta>0$ and $s<\tau$ with

$$
\lim _{t \rightarrow \infty} d\left(\lambda(s, s-t) U_{\eta}(A(s-t)) \mid A(s)\right)=0 .
$$

(iii) The above notions do not depend on the choice of $\tau$, since the behavior of (2.1) on finite time intervals has no effect on the attractivity or repulsivity of a nonautonomous set. This fact is also due to continuity of the general solution.

(iv) In [26, Proposition 2.37], it is shown that past attractors are locally unique, in contrast to past repellers. This uniqueness is due to the fact that convergence is assumed to a fixed fiber.

(v) The Hausdorff semi-distance $d$ in Definition 3.1 can be replaced by the Hausdorff distance $d_{H}$, which for nonempty sets $A, B \subset X$ is defined by $d_{H}(A, B):=\max \{d(A \mid B), d(B \mid A)\}$.

Before introducing domains of attractivity and repulsivity, some auxiliary definitions are needed for the detection of points relative to attractive or repulsive solution which are attracted in forward or backward time, respectively. Given a past attractive solution $\mu:(-\infty, \tau) \rightarrow \mathbb{R}^{N}$, we define

$$
\mathcal{A}_{\mu}^{t}:=\left\{x \in \mathbb{R}^{N}: \lim _{s \rightarrow \infty}\|\lambda(t, t-s)(\mu(t-s)+x)-\mu(t)\|=0\right\} \quad \text { for all } t<\tau,
$$

and for a past repulsive solution $\mu:(-\infty, \tau) \rightarrow \mathbb{R}^{N}$, we define

$$
\mathcal{R}_{\mu}^{t}:=\left\{x \in \mathbb{R}^{N}: \lim _{s \rightarrow \infty}\|\lambda(t-s, t)(\mu(t)+x)-\mu(t-s)\|=0\right\} \text { for all } t<\tau .
$$

The sets $\mathcal{A}_{\mu}^{t}$ and $\mathcal{R}_{\mu}^{t}$ thus represent the allowable perturbations away from the solution $\mu$ that are attracted or repelled, respectively. Note that, due to the continuity of the general solution, the sets $\mathcal{A}_{\mu}^{t}$ are independent of $t$. This is not fulfilled for the sets $\mathcal{R}_{\mu}^{t}$.

For simplicity in description, we want to consider domains of attraction and repulsion as subsets of the phase space.

Definition 3.3 (Domains of attraction and repulsion).

(i) The domain of past attraction of a past attractive solution $\mu:(-\infty, \tau) \rightarrow$ $\mathbb{R}^{N}$ is defined by $\mathcal{A}_{\mu}:=\liminf _{t \rightarrow-\infty} \mathcal{A}_{\mu}^{t}$.

(ii) The domain of past repulsion of a past repulsive solution $\mu:(-\infty, \tau) \rightarrow \mathbb{R}^{N}$ is defined by $\mathcal{R}_{\mu}:=\liminf _{t \rightarrow-\infty} \mathcal{R}_{\mu}^{t}$.

Since the sets $\mathcal{A}_{\mu}^{t}$ are independent of $t$, we have $\mathcal{A}_{\mu}=\mathcal{A}_{\mu}^{t}$ for all $t<\tau$.

We obtain the following complete classification of the attractivity and repulsivity of one-dimensional linear equations.

Example 3.4. Consider the linear nonautonomous differential equation

$$
\dot{x}=a(t) x
$$

with a continuous function $a:(-\infty, 0] \rightarrow \mathbb{R}$. It is easy to see that every invariant and compact nonautonomous set $M \subset(-\infty, 0) \times \mathbb{R}$ is a past attractor if and only if $\lim _{t \rightarrow-\infty} \int_{t}^{0} a(s) d s=-\infty$, and a past repeller if and only if $\lim _{t \rightarrow-\infty} \int_{t}^{0} a(s) d s=$ $\infty$. The domains of attraction and repulsion are equal to $\mathbb{R}$, respectively. 
To study bifurcation phenomena, we consider nonautonomous differential equations of the form

$$
\dot{x}=f(t, x, \alpha),
$$

depending on a real parameter $\alpha$ with a continuous function $f: \Omega \subset \mathbb{R} \times \mathbb{R}^{N} \times \mathbb{R} \rightarrow$ $\mathbb{R}^{N}$. We assume local existence and uniqueness of solutions of (3.1).

Definition 3.5 (Bifurcation and transition). Given a real $\alpha_{0}$, we say that (3.1) admits a supercritical past bifurcation at $\alpha_{0}$ if there exist an $\bar{\alpha}>\alpha_{0}$ and solutions $\mu_{\alpha}$ of (3.1), depending continuously on $\alpha \in\left(\alpha_{0}, \bar{\alpha}\right)$, such that (exactly) one of the following two statements is fulfilled:

(i) $\mu_{\alpha}$ is a past attractive solution of (3.1) for all $\alpha \in\left(\alpha_{0}, \bar{\alpha}\right)$, and

$$
\lim _{\alpha \searrow \alpha_{0}} \sup \left\{\gamma>0: U_{\gamma}(0) \subset \mathcal{A}_{\mu_{\alpha}}\right\}=0
$$

is fulfilled. We call this bifurcation total if $\lim _{\alpha \backslash \alpha_{0}} d\left(\mathcal{A}_{\mu_{\alpha}} \mid\{0\}\right)=0$, otherwise, this bifurcation is called partial, or

(ii) $\mu_{\alpha}$ is a past repulsive solution of (3.1) for all $\alpha \in\left(\alpha_{0}, \bar{\alpha}\right)$, and

$$
\lim _{\alpha \searrow \alpha_{0}} \sup \left\{\gamma>0: U_{\gamma}(0) \subset \mathcal{R}_{\mu_{\alpha}}\right\}=0
$$

is fulfilled. We call this bifurcation total if $\lim _{\alpha \searrow \alpha_{0}} d\left(\mathcal{R}_{\mu_{\alpha}} \mid\{0\}\right)=0$, otherwise, this bifurcation is called partial.

Moreover, we say that (3.1) admits a supercritical past attractor (repeller, respectively) transition at $\alpha_{0}$ if there exist an $\bar{\alpha}>\alpha_{0}$ and past attractors (repellers, respectively) $M_{\alpha}$ of (3.1), $\alpha \in\left(\alpha_{0}, \bar{\alpha}\right)$, with

$$
\lim _{\alpha \searrow \alpha_{0}} \limsup _{t \rightarrow-\infty} \operatorname{diam} M_{\alpha}(t)=0 \text {. }
$$

Accordingly, subcritical past bifurcations and past attractor (repeller, respectively) transitions are defined by considering the limit $\alpha>\alpha_{0}$.

The following nonlinear differential equation is an easy example for the occurrence of a past bifurcation.

Example 3.6. Given a real parameter $\alpha$ and a nonautonomous differential equation

$$
\dot{x}=\alpha a(t) x+b(t) x^{3}=x\left(\alpha a(t)+b(t) x^{2}\right)
$$

with continuous functions $a:(-\infty, 0] \rightarrow \mathbb{R}$ and $b:(-\infty, 0] \rightarrow \mathbb{R}_{\kappa}^{+}$for some $\kappa>0$. We define $w(\alpha, t):=\sqrt{-\alpha a(t) / b(t)}$ for all $t \leq 0$ with $\alpha a(t)<0$. Then, for fixed $t$ with $\alpha a(t)<0$, the zero set of the right hand side is $\{0, \pm w(t)\}$, and for all $t$ with $\alpha a(t) \geq 0$, this zero set is the singleton $\{0\}$. An elementary discussion of the sign of the right hand side of this equation yields that the trivial solution is

(i) past attractive with

$$
\begin{aligned}
& \left(-\liminf _{t \rightarrow-\infty} w(\alpha, t), \liminf _{t \rightarrow-\infty} w(\alpha, t)\right) \subset \mathcal{A}_{0}^{-} \subset\left(-\limsup _{t \rightarrow-\infty} w(\alpha, t), \limsup _{t \rightarrow-\infty} w(\alpha, t)\right) \\
& \quad \text { if } \liminf _{t \rightarrow-\infty}-\alpha a(t) / b(t)>0, \\
& \text { (ii) past repulsive with } \mathcal{R}_{0}^{-}=\mathbb{R} \text { if } \lim _{\operatorname{sim}} \sup _{t \rightarrow-\infty}-\alpha a(t) / b(t) \leq 0 .
\end{aligned}
$$

Since $\lim _{\alpha \rightarrow 0} w(\alpha, t)=0$, this means that (3.2) admits a

(i) supercritical past bifurcation at $\alpha=0$ in case $\liminf _{t \rightarrow-\infty}-a(t) / b(t)>0$ and $\lim \sup _{t \rightarrow-\infty}-a(t) / b(t)<\infty$, 
(ii) subcritical past bifurcation at $\alpha=0$ in case $\liminf _{t \rightarrow-\infty} a(t) / b(t)>0$ and $\lim \sup _{t \rightarrow-\infty} a(t) / b(t)<\infty$.

This example also admits attractor and repeller transitions (see [27, Theorem 6.1]), and note that this differential equation is explicitly solvable, as shown in [19].

\section{Existence of Attractors and Repellers}

In this section, existence results for past attractors and repellers are established. The first lemma implies that past attractors or repellers containing repulsive or attractive solutions, respectively, are nontrivial, i.e., their fibers are not singletons. This corresponds to the well-known situation that the global attractor contains all unstable manifolds (see, e.g., [28, 7] in the autonomous context and [17, 4] for nonautonomous studies).

Lemma 4.1. The following statements are fulfilled:

(i) Let $R \subset(-\infty, \tau) \times \mathbb{R}^{N}$ be a past repeller and $\mu:(-\infty, \tau) \rightarrow \mathbb{R}^{N}$ be a past attractive solution of (2.1) with $\mu(t) \in \operatorname{int} R(t)$ for all $t<\tau$. Then we have

$$
\liminf _{t \rightarrow-\infty}(R(t)-\mu(t)) \supset \mathcal{A}_{\mu}
$$

(ii) Let $A \subset(-\infty, \tau) \times \mathbb{R}^{N}$ be a past attractor and $\mu:(-\infty, \tau) \rightarrow \mathbb{R}^{N}$ be a past repulsive solution of $(2.1)$ with $\mu(t) \in A(t)$ for all $t<\tau$. Then the relation $A(t)-\mu(t) \supset \mathcal{R}_{\mu}^{t}$ holds for all $t<\tau$, and we thus have

$$
\liminf _{t \rightarrow-\infty}(A(t)-\mu(t)) \supset \mathcal{R}_{\mu}
$$

Proof. (i) We choose $x \in \mathcal{A}_{\mu}$ and $\tau^{*}<\tau$. Due to $\mu(t) \in \operatorname{int} R(t)$ for all $t<\tau$, there exists an $\eta>0$ such that $\lambda\left(t, \tau^{*}\right) U_{\eta}\left(\mu\left(\tau^{*}\right)\right) \subset R(t)$ for all $t<\tau$, and since $x \in \mathcal{A}_{\mu}$, there exists a $\tau_{0}<\tau$ with $\lambda\left(\tau^{*}, t\right)(\mu(t)+x) \in U_{\eta}\left(\mu\left(\tau^{*}\right)\right)$ for all $t \leq \tau_{0}$. Hence, we have $\mu(t)+x=\lambda\left(t, \tau^{*}\right) \lambda\left(\tau^{*}, t\right)(\mu(t)+x) \in R(t)$ for all $t \leq \tau_{0}$, and this means that $x \in \liminf _{t \rightarrow-\infty}(R(t)-\mu(t))$.

(ii) Let $t<\tau$, and choose $x \in \mathcal{R}_{\mu}^{t}$ and $\delta>0$ arbitrarily. Since $\mu$ lies in $A$, there exist $\eta>0$ and $\tau_{0}<\tau$ such that $\lambda\left(t, \tau^{*}\right) U_{\eta}\left(\mu\left(\tau^{*}\right)\right) \subset U_{\delta}(A(t))$ for all $\tau^{*} \leq \tau_{0}$. Since $x \in \mathcal{R}_{\mu}^{t}$, there exists a $\tau_{1}<\tau$ with $\lambda\left(\tau^{*}, t\right)(\mu(t)+x) \in U_{\eta}\left(\mu\left(\tau^{*}\right)\right)$ for all $\tau^{*} \leq \tau_{1}$. Hence, with $\tau^{*}:=\min \left\{\tau_{0}, \tau_{1}\right\}$, the relation

$$
\mu(t)+x=\lambda\left(t, \tau^{*}\right) \lambda\left(\tau^{*}, t\right)(\mu(t)+x) \in U_{\delta}(A(t))
$$

holds. Since $\delta$ was chosen arbitrarily and $A(t)$ is compact, we have $\mu(t)+x \in A(t)$, and thus, $\mu(t)+\mathcal{R}_{\mu}^{t} \subset A(t)$ is fulfilled.

The following existence result for pullback attractors (cf. Remark 3.2) is needed in the proof of Theorem 4.3 below. A proof can be found, e.g., in [10, Theorem $3.5]$.

Theorem 4.2 (Existence of pullback attractors). Consider a collection of nonautonomous sets $\mathcal{D}$, and let $B \subset(-\infty, \tau) \times \mathbb{R}^{N}$ be a compact pullback absorbing set w.r.t. $\mathcal{D}$, i.e., all fibers of $B$ are compact and for all $D \in \mathcal{D}$ and $\tau^{*} \leq \tau$, there exists a $t^{*}<\tau^{*}$ such that $\lambda\left(\tau^{*}, t\right) D(t) \subseteq B\left(\tau^{*}\right)$ for all $t \leq t^{*}$. Then there exists a pullback attractor $A$ with attraction universe $\mathcal{D}$, which fulfills the representation

$$
A\left(\tau^{*}\right)=\bigcap_{t^{*} \leq \tau^{*}} \operatorname{cls} \bigcup_{t \leq t^{*}} \lambda\left(\tau^{*}, t\right) B(t) \quad \text { for all } \tau^{*}<\tau .
$$


If, in addition, $A \in \mathcal{D}$, then $A$ is uniquely determined. In case $B \in \mathcal{D}$, the relation $A \subseteq B$ is fulfilled.

In the following theorem, sufficient conditions are stated to guarantee the existence of a past attractor or past repeller which contains a past repulsive or past attractive solution, respectively. These conditions contain statements about the dynamical behavior at the boundary of the domain of attraction or repulsion of the attractive or repulsive solution, respectively.

Theorem 4.3 (Existence of past attractors and past repellers). The following statements are fulfilled:

(i) Suppose that $\mu:(-\infty, \tau) \rightarrow \mathbb{R}^{N}$ is a past attractive solution of $(2.1)$ such that $\mathcal{A}_{\mu}$ is bounded and there exist $\varepsilon>0$ and $\tau_{0}<\tau$ with

$$
\lim _{t \rightarrow-\infty} d\left(\lambda\left(t, \tau^{*}\right) U_{\varepsilon}\left(\mu\left(\tau^{*}\right)+\mathcal{A}_{\mu}\right) \mid \mu(t)+\mathcal{A}_{\mu}\right)=0 \quad \text { for all } \tau^{*} \leq \tau_{0} .
$$

Then there exists a $\beta>0$ such that the invariant and compact past nonautonomous set $R$, defined by $R(t):=\lambda\left(t, \tau_{0}\right) \operatorname{cls} U_{\beta}\left(\mu\left(\tau_{0}\right)\right)$ for all $t \leq \tau_{0}$, is a past repeller fulfilling

$$
\mathcal{A}_{\mu} \subset \liminf _{t \rightarrow-\infty}(R(t)-\mu(t)) \subset \limsup _{t \rightarrow-\infty}(R(t)-\mu(t)) \subset \operatorname{cls} \mathcal{A}_{\mu} .
$$

(ii) Suppose that $\mu:(-\infty, \tau) \rightarrow \mathbb{R}^{N}$ is a past repulsive solution of $(2.1)$ such that $\mathcal{R}_{\mu}$ is bounded and there exists an $\eta>0$ such that for all $\varepsilon>0$, there exists a $\tau_{0}<\tau$ such that for all $\tau^{*} \leq \tau_{0}$, there is a $T>0$ with

$\lambda\left(\tau^{*}, \tau^{*}-t\right) U_{\eta}\left(\mu\left(\tau^{*}-t\right)+\mathcal{R}_{\mu}\right) \subset U_{\varepsilon}\left(\mu\left(\tau^{*}\right)+\mathcal{R}_{\mu}\right) \quad$ for all $t \geq T$.

Then there exists a past attractor $A \subset(-\infty, \tau) \times \mathbb{R}^{N}$ fulfilling

$$
\mathcal{R}_{\mu} \subset \liminf _{t \rightarrow-\infty}(A(t)-\mu(t)) \subset \limsup _{t \rightarrow-\infty}(A(t)-\mu(t)) \subset \operatorname{cls} \mathcal{R}_{\mu} .
$$

Proof. (i) We choose a $\beta>0$ with $\operatorname{cls} U_{\beta}(0) \subset U_{\varepsilon}\left(\mathcal{A}_{\mu}\right)$ and define $R(t):=$ $\lambda\left(t, \tau_{0}\right)$ cls $U_{\beta}\left(\mu\left(\tau_{0}\right)\right)$ for all $t \leq \tau_{0}$. This means that

$$
R(t) \subset \lambda\left(t, \tau_{0}\right) U_{\varepsilon}\left(\mu\left(\tau_{0}\right)+\mathcal{A}_{\mu}\right) \quad \text { for all } t \leq \tau_{0} .
$$

Moreover,

$$
\lim _{t \rightarrow-\infty} d\left(R(t) \mid \mu(t)+\mathcal{A}_{\mu}\right) \stackrel{(4.2)}{\leq} \lim _{t \rightarrow-\infty} d\left(\lambda\left(t, \tau_{0}\right) U_{\varepsilon}\left(\mu\left(\tau_{0}\right)+\mathcal{A}_{\mu}\right) \mid \mu(t)+\mathcal{A}_{\mu}\right) \stackrel{(4.1)}{=} 0
$$

is fulfilled, and thus, $\lim \sup _{t \rightarrow-\infty}(R(t)-\mu(t)) \subset \operatorname{cls} \mathcal{A}_{\mu}$ holds. Next, we show that $R$ is a past repeller. First, suppose to the contrary that

$$
\delta:=\liminf _{t \rightarrow-\infty} d\left(\mu(t)+\mathcal{A}_{\mu} \mid R(t)\right)>0 .
$$

Since cls $\mathcal{A}_{\mu}$ is compact, there exist an $n \in \mathbb{N}$ and $x_{1}, \ldots, x_{n} \in \operatorname{cls} \mathcal{A}_{\mu}$ such that $\operatorname{cls} \mathcal{A}_{\mu} \subset \cup_{i=1}^{n} U_{\delta / 4}\left(x_{i}\right)$. For each $i \in\{1, \ldots, n\}$, we choose arbitrary elements $y_{i} \in U_{\delta / 4}\left(x_{i}\right) \cap \mathcal{A}_{\mu}$. Then the set $C:=\left\{y_{1}, \ldots, y_{n}\right\}$ is a subset of $\mathcal{A}_{\mu}$ with

$$
d\left(\mathcal{A}_{\mu} \mid C\right) \leq \frac{\delta}{2}
$$

Since $C$ is finite, there exists a $\tau_{1} \leq \tau_{0}$ with $\lambda\left(\tau_{0}, t\right)(\mu(t)+C) \subset U_{\beta}\left(\mu\left(\tau_{0}\right)\right) \subset$ $R\left(\tau_{0}\right)$ for all $t \leq \tau_{1}$. This means that, due to the invariance of $R$, we obtain 
$\lim _{t \rightarrow-\infty} d(\mu(t)+C \mid R(t))=0$. This implies

$$
\begin{aligned}
& \liminf _{t \rightarrow-\infty} d\left(\mu(t)+\mathcal{A}_{\mu} \mid R(t)\right) \\
\leq & \liminf _{t \rightarrow-\infty}\left(d\left(\mu(t)+\mathcal{A}_{\mu} \mid \mu(t)+C\right)+d(\mu(t)+C \mid R(t))\right) \stackrel{(4.4)}{\leq} \frac{\delta}{2} .
\end{aligned}
$$

This is a contradiction to (4.3), and thus, we have

$$
\lim _{t \rightarrow-\infty} d\left(\mu(t)+\mathcal{A}_{\mu} \mid R(t)\right)=0 .
$$

Furthermore, there exists a $\tau_{2} \leq \tau_{0}$ with

$$
\begin{aligned}
& \quad d\left(R\left(\tau^{*}\right) \mid \mu\left(\tau^{*}\right)+\mathcal{A}_{\mu}\right) \\
& \stackrel{(4.2)}{\leq} d\left(\lambda\left(\tau^{*}, \tau_{0}\right) U_{\varepsilon}\left(\mu\left(\tau_{0}\right)+\mathcal{A}_{\mu}\right) \mid \mu\left(\tau^{*}\right)+\mathcal{A}_{\mu}\right) \stackrel{(4.1)}{<} \frac{\varepsilon}{2} \quad \text { for all } \tau^{*} \leq \tau_{2} .
\end{aligned}
$$

Hence, we have

(4.6) $U_{\varepsilon}\left(\mu\left(\tau^{*}\right)+\mathcal{A}_{\mu}\right) \supset U_{\varepsilon / 2}\left(U_{\varepsilon / 2}\left(\mu\left(\tau^{*}\right)+\mathcal{A}_{\mu}\right)\right) \supset U_{\varepsilon / 2}\left(R\left(\tau^{*}\right)\right)$ for all $\tau^{*} \leq \tau_{2}$, and for all $\tau^{*} \leq \tau_{2}$, the inequality

$$
\begin{array}{ll} 
& \lim _{t \rightarrow-\infty} d\left(\lambda\left(t, \tau^{*}\right) U_{\varepsilon / 2}\left(R\left(\tau^{*}\right)\right) \mid R(t)\right) \\
\stackrel{(4.6)}{\leq} & \lim _{t \rightarrow-\infty} d\left(\lambda\left(t, \tau^{*}\right) U_{\varepsilon}\left(\mu\left(\tau^{*}\right)+\mathcal{A}_{\mu}\right) \mid R(t)\right) \\
\leq & \lim _{t \rightarrow-\infty} d\left(\lambda\left(t, \tau^{*}\right) U_{\varepsilon}\left(\mu\left(\tau^{*}\right)+\mathcal{A}_{\mu}\right) \mid \mu(t)+\mathcal{A}_{\mu}\right)+\lim _{t \rightarrow-\infty} d\left(\mu(t)+\mathcal{A}_{\mu} \mid R(t)\right) \\
\stackrel{(4.1),(4.5)}{=} & 0
\end{array}
$$

holds. Thus, $R$ is a past repeller. The relation $\liminf \inf _{t \rightarrow-\infty}(R(t)-\mu(t)) \supset \mathcal{A}_{\mu}$ follows from Lemma 4.1 (i).

(ii) We define the nonautonomous set $M$ by its fibers $M(t):=U_{\eta}\left(\mu(t)+\mathcal{R}_{\mu}\right)$ for all $t<\tau$. Due to the hypotheses, the fibers of a compact pullback absorbing set $B$ w.r.t. $\{M\}$ can be defined with the following property: For all $\varepsilon>0$, there exists a $\tau^{*}<\tau$ such that

$$
\mathcal{R}_{\mu}+\mu(t) \subset B(t) \subset U_{\varepsilon}\left(\mathcal{R}_{\mu}+\mu(t)\right) \text { for all } t \leq \tau^{*}
$$

Thus, Theorem 4.2 implies the existence of a pullback attractor $A \subset B$ fulfilling

$$
\limsup _{t \rightarrow-\infty}(A(t)-\mu(t)) \subset \limsup _{t \rightarrow-\infty}(B(t)-\mu(t)) \stackrel{(4.7)}{\subset} \operatorname{cls} \mathcal{R}_{\mu} .
$$

$A$ is also a past attractor (cf. Remark $3.2(\mathrm{i})$ ). The relation $\lim \inf _{t \rightarrow-\infty}(A(t)-$ $\mu(t)) \supset \mathcal{R}_{\mu}$ follows from Lemma 4.1 (ii).

\section{Basic Properties of Asymptotically Autonomous Systems}

In this section, some useful lemmata are derived for asymptotically autonomous differential equations. We want to pose conditions on the asymptotically autonomous system in order to establish relationships to the limiting system, and we study in particular typical situations which appear in presence of autonomous bifurcations. 
The first lemma deals with the question of controlling the distances of the time evolutions of both systems. We consider an open and convex set $D \subset \mathbb{R}^{N}$, a nonautonomous differential equation

$$
\dot{x}=f(t, x)
$$

with a $C^{1}$-function $f:(-\infty, 0) \times D \rightarrow \mathbb{R}^{N}$ and an autonomous differential equation

$$
\dot{x}=g(x)
$$

with a $C^{1}$-function $g: D \rightarrow \mathbb{R}^{N}$. We assume that

$$
\lim _{t \rightarrow-\infty} f(t, x)=g(x) \quad \text { uniformly for } x \in D .
$$

The general solution of (5.1) is denoted by $\lambda$, and let $\varphi$ be the flow of (5.2).

Lemma 5.1. Given a compact and convex set $K \subset D$, the following statements hold:

(i) For all $T>0$ and $\varepsilon>0$, there exists a $\tau_{0}<-T$ such that for all $T^{\prime} \leq T$ and $x \in K$ with $\varphi(t, x) \in K$ for all $t \in\left[0, T^{\prime}\right]$, we have

$$
\|\lambda(\tau+t, \tau) x-\varphi(t, x)\| \leq \varepsilon \quad \text { for all } \tau \leq \tau_{0} \text { and } t \in\left[0, T^{\prime}\right] .
$$

(ii) For all $T>0$ and $\varepsilon>0$, there exists a $\tau_{0}<0$ such that for all $T^{\prime} \leq T$ and $x \in K$ with $\varphi(-t, x) \in K$ for all $t \in\left[0, T^{\prime}\right]$, we have

$$
\|\lambda(\tau-t, \tau) x-\varphi(-t, x)\| \leq \varepsilon \quad \text { for all } \tau \leq \tau_{0} \text { and } t \in\left[0, T^{\prime}\right] .
$$

Proof. (i) Since $D$ is open, there exist a compact and convex set $\bar{K}$ and an $\eta>0$ such that $U_{\eta}(K) \subset \bar{K}$. We choose $T>0$ and $\varepsilon>0$ arbitrarily and define $M:=$ $\max _{x \in \bar{K}}\|D g(x)\|$. Due to (5.3), there exists a $\tau_{0}<-T$ with

$$
\|f(t+T, x)-g(x)\| \leq \frac{\min \{\varepsilon, \eta\}}{T e^{M T}} \text { for all } t \leq \tau_{0} \text { and } x \in D .
$$

For the rest of this proof, we fix arbitrary numbers $\tau \leq \tau_{0}, T^{\prime} \leq T$ and $x \in K$ fulfilling $\varphi(t, x) \in K$ for all $t \in\left[0, T^{\prime}\right]$. Since

$\lambda(t+\tau, \tau) x-\varphi(t, x)=\int_{0}^{t}(f(s+\tau, \lambda(s+\tau, \tau) x)-g(\varphi(s, x))) d s$ for all $t \in\left[0, T^{\prime}\right]$,

it follows from the mean value inequality (see, e.g., [1, Theorem 2.4.8, p. 87]) that

$$
\begin{aligned}
\|\lambda(t+\tau, \tau) x-\varphi(t, x)\| & \leq \int_{0}^{t}\|f(s+\tau, \lambda(s+\tau, \tau) x)-g(\varphi(s, x))\| d s \\
& \leq \int_{0}^{t}(\|f(s+\tau, \lambda(s+\tau, \tau) x)-g(\lambda(s+\tau, \tau) x)\|+ \\
& \left\|\frac{t \min \{\varepsilon, \eta\}}{T e^{M T}}+M \int_{0}^{t}\right\| \lambda(s+\tau, \tau) x-\varphi(s, x) \| d s .
\end{aligned}
$$

Assume, there exists a $t \in\left(0, T^{\prime}\right)$ with $\|\lambda(t+\tau, \tau) x-\varphi(t, x)\| \geq \min \{\varepsilon, \eta\}$. We define

$$
T^{*}:=\min \left\{t \in\left(0, T^{\prime}\right):\|\lambda(t+\tau, \tau) x-\varphi(t, x)\| \geq \min \{\varepsilon, \eta\}\right\}<T^{\prime} .
$$

Hence, from Gronwall's inequality (see, e.g., [1, Theorem 4.1.7, p. 242]), we obtain

$$
\left\|\lambda\left(T^{*}+\tau, \tau\right) x-\varphi\left(T^{*}, x\right)\right\| \leq \frac{T^{*} \min \{\varepsilon, \eta\}}{T e^{M T}} e^{M T^{*}}<\min \{\varepsilon, \eta\} .
$$


This is a contradiction and finishes the proof of this lemma.

(ii) See proof of (i).

In case of the classical autonomous bifurcations for ordinary differential equations (such as pitchfork, transcritical, saddle node and Hopf bifurcation), after the bifurcation, the phase space can be separated into three invariant parts. We therefore restrict attention to the following situation: The domain $D \subset \mathbb{R}^{N}$ of the autonomous equation (5.2) is the disjoint union of

- an open set $S^{i}$ (inner area),

- an open set $S^{\circ}$ (outer area),

- a compact set $S=\partial S^{i}=\partial S^{o}$ with int $S=\emptyset$.

(a)

(b)

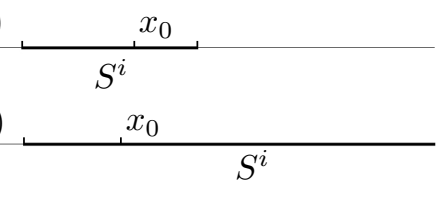

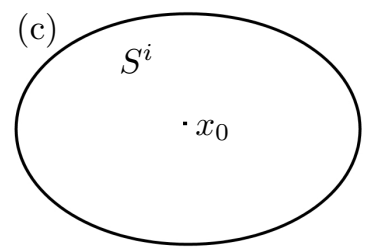

Figure 4. The above situation in case of a (a) pitchfork bifurcation,

(b) transcritical or saddle node bifurcation, (c) Hopf bifurcation.

The occurrence of one of the above mentioned autonomous bifurcations means that (exactly) one of the following two hypotheses holds:

- Hypothesis $\left(\mathrm{H}_{1}\right)$ :

(i) The inner area $S^{i}$ is forward invariant, i.e., $\varphi(t, x) \in S^{i}$ for all $t \geq 0$ and $x \in S^{i}$, and there exists an attractive equilibrium $x_{0} \in S^{i}$ such that $\lim _{t \rightarrow \infty} d\left(\varphi(t, K) \mid\left\{x_{0}\right\}\right)=0$ for all compact sets $K \subset S^{i}$.

(ii) The outer area $S^{o}$ is backward invariant, i.e., $\varphi(t, x) \in S^{o}$ for all $t \leq 0$ and $x \in S^{o}$, and $S$ is a repeller, i.e., there exists an $\eta>0$ such that $\lim _{t \rightarrow \infty} d\left(\varphi\left(-t, U_{\eta}(S)\right) \mid S\right)=0$.

(iii) $S$ is invariant, i.e., $\varphi(t, x) \in S$ for all $t \in \mathbb{R}$ and $x \in S$.

- Hypothesis $\left(\mathrm{H}_{2}\right)$ :

(i) The inner area $S^{i}$ is backward invariant, and there exists a repulsive equilibrium $x_{0} \in S^{i}$ such that $\lim _{t \rightarrow \infty} d\left(\varphi(-t, K) \mid\left\{x_{0}\right\}\right)=0$ for all compact sets $K \subset S^{i}$.

(ii) The outer area $S^{o}$ is forward invariant, and $S$ is an attractor, i.e., there exists an $\eta>0$ with $\lim _{t \rightarrow \infty} d\left(\varphi\left(t, U_{\eta}(S)\right) \mid S\right)=0$.

(iii) $S$ is invariant.

Note that in case of global existence of solutions of (5.2), the invariance of $S$ implies invariance of $S^{i}$ and $S^{o}$, and thus, the parts (i) and (ii) can be dropped.

Lemma 5.2. Under Hypothesis $\left(H_{1}\right)$, the following statements hold:

(i) For all $\delta>0$, there exists a $T>0$ such that for all $x \in D$ with $d(x, S) \geq \delta$, there exists a $\bar{T} \in[0, T]$ with $d(\varphi(\bar{T}, x), S) \geq \eta$.

(ii) For all $\gamma>0$, there exists a $\delta>0$ with $\varphi\left(-t, U_{\delta}\left(S^{i}\right)\right) \subset U_{\gamma}\left(S^{i}\right)$ for all $t \geq 0$.

Similar statements are fulfilled in case of Hypothesis $\left(\mathrm{H}_{2}\right)$.

Proof. (i) We choose $\delta>0$ arbitrarily. Due to the hypotheses, there exists a $T>0$ with $\varphi\left(-T, U_{\eta}(S)\right) \subset U_{\delta / 2}(S)$. This implies the assertion. 
(ii) We choose $\gamma>0$ arbitrarily. Since $S$ is repulsive and $S=\partial S^{i}$, there exists a $T>0$ with $\varphi\left(-t, U_{\eta}\left(S^{i}\right)\right) \subset U_{\gamma}\left(S^{i}\right)$ for all $t>T$. Arguing negatively, we assume that for all $n \in \mathbb{N}$, there exist $t_{n} \in[0, T]$ and $x_{n} \in U_{1 / n}\left(S^{i}\right)$ with $d\left(\varphi\left(-t_{n}, x_{n}\right), S^{i}\right)=$ $d\left(\varphi\left(-t_{n}, x_{n}\right), S\right) \geq \gamma$. Since $S$ is compact, we assume w.l.o.g. that the sequence $\left\{x_{n}\right\}_{n \in \mathbb{N}}$ is convergent with limit $x \in S$. Due to the continuity of the flow $\varphi$ and the invariance of $S$, there exists a $\beta>0$ such that for all $y \in U_{\beta}(x)$ and $t \in[0, T]$, we have $d(\varphi(-t, y), S)<\gamma / 2$. This is a contradiction and finishes the proof of this lemma.

Remark 5.3. In case the outer area is forward invariant, statement (i) of the above lemma can be simplified as follows: For all $\delta>0$, there exists a $T>0$ such that for all $x \in D$ with $d(x, S) \geq \delta$, the relation $d(\varphi(T, x), S) \geq \eta$ is fulfilled.

The following lemma provides a first example that the behavior of the limiting autonomous system is inherited by the asymptotically autonomous system. In particular, the question of determining past attraction and repulsion areas is treated.

Lemma 5.4. The following statements hold:

(i) We suppose that Hypothesis $\left(H_{1}\right)$ is fulfilled, and let $\mu:(-\infty, \tau) \rightarrow \mathbb{R}^{N}$ be a past attractive solution of (5.1) with $\lim _{t \rightarrow-\infty} \mu(t)=x_{0}$. Then we have

$$
\operatorname{int} \mathcal{A}_{\mu}=S^{i}-x_{0} \text {. }
$$

If, in addition, $S^{i}$ is bounded, then there exist $s<\tau$ and a past repeller $R \subset(-\infty, s) \times D$ with

$$
S^{i} \subset \liminf _{t \rightarrow-\infty} R(t) \subset \limsup _{t \rightarrow-\infty} R(t) \subset \operatorname{cls} S^{i} .
$$

(ii) We suppose that Hypothesis $\left(H_{2}\right)$ is fulfilled, and let $\mu:(-\infty, \tau) \rightarrow \mathbb{R}^{N}$ be a past repulsive solution of (5.1) with $\lim _{t \rightarrow-\infty} \mu(t)=x_{0}$. Then we have

$$
\operatorname{int} \mathcal{R}_{\mu}=S^{i}-x_{0}
$$

If, in addition, $S^{i}$ is bounded, then there exist $s<\tau$ and a past attractor $A \subset(-\infty, s) \times D$ with

$$
S^{i} \subset \liminf _{t \rightarrow-\infty} A(t) \subset \limsup _{t \rightarrow-\infty} A(t) \subset \operatorname{cls} S^{i} .
$$

Proof. (i) First, assume w.l.o.g. that $x_{0}=0$. The remaining proof is divided into four steps.

Step 1. int $\mathcal{A}_{\mu} \supset S^{i}$.

Since $\mu$ is past attractive, there exists a $\gamma>0$ such that

$$
\lim _{t \rightarrow \infty} d\left(\lambda(s, s-t) U_{\gamma}(\mu(s-t)) \mid \mu(s)\right)=0 \text { for all } s<\tau .
$$

We choose $y \in S^{i}$ arbitrarily. Let $\zeta>0$ such that there exists a $\delta>0$ with $\operatorname{cls} U_{\delta}\left(U_{\zeta}(y)\right) \subset S^{i}$. Because of $\lim _{t \rightarrow-\infty} \mu(t)=0$, there exists a $t_{1}<\tau$ such that $\mu(t) \in U_{\min \{\gamma / 3, \delta\}}(0)$ for all $t \leq t_{1}$. Due to the attractivity of $x_{0}=0$, there exists a $T>0$ such that $d\left(\varphi\left(T, U_{\delta}\left(U_{\zeta}(y)\right)\right) \mid\{0\}\right)<\gamma / 3$. Since it is possible to choose a compact and convex superset $K$ of $S^{i} \subset D$ ( $D$ is convex), Lemma 5.1 (i) implies that there exists a $t_{2}<t_{1}-T$ with

$$
\|\lambda(t+T, t) x-\varphi(T, x)\| \leq \frac{\gamma}{3} \quad \text { for all } t \leq t_{2} \text { and } x \in \underbrace{\mu(t)+U_{\zeta}(y)}_{\subset U_{\delta}\left(U_{\zeta}(y)\right)} .
$$


Hence, for all $t \leq t_{2}$ and $x \in \mu(t)+U_{\zeta}(y)$, we have

$$
\begin{aligned}
\|\lambda(t+T, t) x-\mu(t+T)\| & \leq\|\lambda(t+T, t) x-\varphi(T, x)\|+\|\varphi(T, x)\|+\|\mu(t+T)\| \\
& <\frac{\gamma}{3}+\frac{\gamma}{3}+\frac{\gamma}{3}=\gamma .
\end{aligned}
$$

Thus,

$$
\begin{aligned}
& \lim _{t \rightarrow-\infty} d\left(\lambda\left(t_{2}, t\right)\left(\mu(t)+U_{\zeta}(y)\right) \mid\left\{\mu\left(t_{2}\right)\right\}\right) \\
= & \lim _{t \rightarrow-\infty} d(\lambda\left(t_{2}, t+T\right) \underbrace{\lambda(t+T, t)\left(\mu(t)+U_{\zeta}(y)\right)}_{\subset U_{\gamma}(\mu(t+T))} \mid\left\{\mu\left(t_{2}\right)\right\}) \stackrel{(5.4)}{=} 0 .
\end{aligned}
$$

This implies $y \in \operatorname{int} \mathcal{A}_{\mu}$, and hence, int $\mathcal{A}_{\mu} \supset S^{i}$.

Step 2. int $\mathcal{A}_{\mu} \subset S^{i}$.

We choose $y \in S^{o}$ and $\beta>0$ such that $U_{\beta}(0) \subset S^{i}$ and define $\delta:=d(y, S)>0$. Because of Lemma 5.2 (i), there exists a $T>0$ such that for all $x \in S^{o}$ with $d(x, S) \geq \min \{\delta / 2, \eta / 3\}$ (recall that $\eta$ is determined by Hypothesis $\left(\mathrm{H}_{1}\right)$ ), there exists a $\overline{\bar{T}} \in[0, T]$ with

$$
d(\varphi(\bar{T}, x), S) \geq \eta \quad \text { and } \quad \varphi(\bar{T}, x) \in S^{o} .
$$

Moreover, there exists a $t_{1}<\tau$ with $\mu(t) \in U_{\min \{\delta / 2, \eta / 3, \beta / 4\}}(0) \subset S^{i}$ for all $t \leq t_{1}$. Let $K$ be a compact and convex superset of $U_{\eta}(S)$. Then, due to Lemma 5.1 (i), there exists a $t_{2}<t_{1}$ such that for all $\hat{T} \in[0, T]$ and $x \in K$ with $\varphi(t, x) \in K$ for all $t \in[0, \hat{T}]$, we have

$$
\|\lambda(\bar{t}+t, \bar{t}) x-\varphi(t, x)\| \leq \min \left\{\frac{\beta}{2}, \frac{\eta}{3}\right\} \quad \text { for all } \bar{t} \leq t_{2} \text { and } t \in[0, \hat{T}] .
$$

We argue negatively and suppose that $\lim _{t \rightarrow-\infty}\left\|\lambda\left(t_{2}, t\right)(y+\mu(t))-\mu\left(t_{2}\right)\right\|=0$. Since $\mu\left(t_{2}\right) \in U_{\beta / 4}(0)$, this implies that there exists a $t_{3}<t_{2}$ with

$$
\lambda\left(t_{2}, t_{3}\right)\left(y+\mu\left(t_{3}\right)\right) \in U_{\frac{\beta}{2}}(0) .
$$

We define

$$
\begin{array}{r}
s:=\max \left\{t \in\left[t_{3}, t_{2}\right]: d\left(\lambda\left(t, t_{3}\right)\left(y+\mu\left(t_{3}\right)\right), S\right) \geq \min \{\eta / 2, \delta / 2\}\right. \\
\text { and } \left.\lambda\left(t, t_{3}\right)\left(y+\mu\left(t_{3}\right)\right) \in S^{o}\right\} .
\end{array}
$$

This implies $d\left(\lambda\left(s, t_{3}\right)\left(y+\mu\left(t_{3}\right)\right), S\right)=\min \left\{\frac{\eta}{2}, \frac{\delta}{2}\right\}$ and $\lambda\left(s, t_{3}\right)\left(y+\mu\left(t_{3}\right)\right) \in S^{o}$. We distinguish two cases.

Case 1. $t_{2}-s \leq T$.

Case 1.1. For all $t \in\left[0, t_{2}-s\right]$, we have $\varphi\left(t, \lambda\left(s, t_{3}\right)\left(y+\mu\left(t_{3}\right)\right)\right) \in K$.

Due to (5.6), we have

$$
\left\|\varphi\left(t, \lambda\left(s, t_{3}\right)\left(y+\mu\left(t_{3}\right)\right)\right)-\lambda\left(t+s, t_{3}\right)\left(y+\mu\left(t_{3}\right)\right)\right\| \leq \frac{\beta}{2} \quad \text { for all } t \in\left[0, t_{2}-s\right] .
$$

Since $\left\|\varphi\left(t, \lambda\left(s, t_{3}\right)\left(y+\mu\left(t_{3}\right)\right)\right)\right\| \geq \beta$ for all $t \in\left[0, t_{2}-s\right]\left(\right.$ note that $\left.U_{\beta}(0) \subset S^{i}\right)$, this leads to

$$
\begin{aligned}
\left\|\lambda\left(t_{2}, t_{3}\right)\left(y+\mu\left(t_{3}\right)\right)\right\| \geq & \left\|\varphi\left(t_{2}-s, \lambda\left(s, t_{3}\right)\left(y+\mu\left(t_{3}\right)\right)\right)\right\|- \\
& \left\|\lambda\left(t_{2}, t_{3}\right)\left(y+\mu\left(t_{3}\right)\right)-\varphi\left(t_{2}-s, \lambda\left(s, t_{3}\right)\left(y+\mu\left(t_{3}\right)\right)\right)\right\| \\
\geq & \beta-\frac{\beta}{2}=\frac{\beta}{2},
\end{aligned}
$$


which contradicts (5.7).

Case 1.2. There exists a $\bar{t} \in\left[0, t_{2}-s\right]$ with $\varphi\left(\bar{t}, \lambda\left(s, t_{3}\right)\left(y+\mu\left(t_{3}\right)\right)\right) \notin K$.

By defining $\bar{s}:=\inf \left\{t \in\left[0, t_{2}-s\right]: \varphi\left(t, \lambda\left(s, t_{3}\right)\left(y+\mu\left(t_{3}\right)\right)\right) \notin K\right\}>0$, we obtain $d\left(\varphi\left(\bar{s}, \lambda\left(s, t_{3}\right)\left(y+\mu\left(t_{3}\right)\right)\right), S\right) \geq \eta$. Due to $(5.6)$, we have

$$
\left\|\lambda\left(\bar{s}+s, t_{3}\right)\left(y+\mu\left(t_{3}\right)\right)-\varphi\left(\bar{s}, \lambda\left(s, t_{3}\right)\left(y+\mu\left(t_{3}\right)\right)\right)\right\| \leq \frac{\eta}{3},
$$

and thus, $d\left(\lambda\left(\bar{s}+s, t_{3}\right)\left(y+\mu\left(t_{3}\right)\right), S\right) \geq \frac{2 \eta}{3}$ and $\lambda\left(\bar{s}+s, t_{3}\right)\left(y+\mu\left(t_{3}\right)\right) \in S^{o}$ hold. This is a contradiction to the definition of $s$.

Case 2. $t_{2}-s>T$.

Case 2.1. For all $t \in[0, T]$, we have $\varphi\left(t, \lambda\left(s, t_{3}\right)\left(y+\mu\left(t_{3}\right)\right)\right) \in K$.

Because of $(5.5)$ and $d\left(\lambda\left(s, t_{3}\right)\left(y+\mu\left(t_{3}\right)\right), S\right)=\min \left\{\frac{\delta}{2}, \frac{\eta}{2}\right\} \geq \min \left\{\frac{\delta}{2}, \frac{\eta}{3}\right\}$, there exists a $\bar{T} \in[0, T]$ with $d\left(\varphi\left(\bar{T}, \lambda\left(s, t_{3}\right)\left(y+\mu\left(t_{3}\right)\right)\right), S\right) \geq \eta$, and (5.6) yields

$$
\left\|\varphi\left(\bar{T}, \lambda\left(s, t_{3}\right)\left(y+\mu\left(t_{3}\right)\right)\right)-\lambda\left(\bar{T}+s, t_{3}\right)\left(y+\mu\left(t_{3}\right)\right)\right\| \leq \frac{\eta}{3} .
$$

Together, this implies $d\left(\lambda\left(\bar{T}+s, t_{3}\right)\left(y+\mu\left(t_{3}\right)\right), S\right) \geq 2 / 3 \eta$ and $\lambda\left(\bar{T}+s, t_{3}\right)(y+$ $\left.\mu\left(t_{3}\right)\right) \in S^{o}$. This is a contradiction to the definition of $s$.

Case 2.2. There exists a $\bar{t} \in[0, T]$ with $\varphi\left(\bar{t}, \lambda\left(s, t_{3}\right)\left(y+\mu\left(t_{3}\right)\right)\right) \notin K$.

This case is treated analogously to Case 1.2 (by writing $T$ instead of $t_{2}-s$ ).

Consequently, we have $y \notin \mathcal{A}_{\mu}$. This leads to the assertion, since int $S=\emptyset$.

Step 3. For all $\kappa \leq \eta$, there exist $T>0$ and $t_{1}<\tau$ such that for all $t_{2}<t_{1}$ and $t>T$, we have

$$
\lambda\left(t_{2}-t, t_{2}\right) U_{5 \kappa / 6}\left(\mathcal{A}_{\mu}+\mu\left(t_{2}\right)\right) \subset U_{2 \kappa / 3}\left(\mathcal{A}_{\mu}+\mu\left(t_{2}-t\right)\right) .
$$

We choose $\kappa \leq \eta$ arbitrarily. By applying Lemma 5.2 (ii), there exists a $\delta>0$ with

$$
\varphi\left(-t, U_{\delta}\left(S^{i}\right)\right) \subset U_{\kappa / 4}\left(S^{i}\right) \text { for all } t \geq 0 .
$$

Due to the repulsivity of $S$, there exists a $T>0$ with $\varphi\left(-t, U_{\kappa}\left(S^{i}\right)\right) \subset$ $U_{\delta / 2}\left(S^{i}\right)$ for all $t>T$. By choosing $K$ as a convex and compact superset of $\cup_{t \in[0, T]} \varphi\left(-t, U_{\kappa}\left(S^{i}\right)\right)$, we can apply Lemma 5.1 (ii), and we therefore get a $t_{1}<\tau$ with

$$
\begin{aligned}
& \left\|\lambda\left(t_{2}-t, t_{2}\right) x-\varphi(-t, x)\right\| \leq \frac{\delta}{2} \quad \text { for all } x \in U_{\kappa}\left(S^{i}\right), t_{2}<t_{1}, t \in[0, T] \\
& \text { and } \quad\|\mu(t)\| \leq \frac{\kappa}{6} \quad \text { for all } t<t_{1} \text {. }
\end{aligned}
$$

Thus,

$$
\lambda\left(t_{2}-T, t_{2}\right) U_{\kappa}\left(S^{i}\right) \subset U_{\delta}\left(S^{i}\right) \subset U_{\kappa / 4}\left(S^{i}\right) \text { for all } t_{2}<t_{1}
$$

is fulfilled. Because of (5.9), this leads to $\varphi\left(-t, \lambda\left(t_{2}-T, t_{2}\right) U_{\kappa}\left(S^{i}\right)\right) \subset U_{\kappa / 4}\left(S^{i}\right)$ for all $t_{2}<t_{1}$ and $t \geq 0$. Due to (5.10) and $\delta / 2<\kappa / 4$, we have $\lambda\left(t_{2}-t, t_{2}\right) U_{\kappa}\left(S^{i}\right) \subset$ $U_{\kappa / 2}\left(S^{i}\right)$ for all $t_{2}<t_{1}$ and $t \in[T, 2 T]$. Suppose now, there exist $\bar{t}>2 T$ and $\bar{t}_{2}<t_{1}$ with $d\left(\lambda\left(\bar{t}_{2}-\bar{t}, \bar{t}_{2}\right) U_{\kappa}\left(S^{i}\right) \mid S^{i}\right) \geq \kappa / 2$. We define

$$
s:=\inf \left\{t>2 T: d\left(\lambda\left(\bar{t}_{2}-t, \bar{t}_{2}\right) U_{\kappa}\left(S^{i}\right) \mid S^{i}\right) \geq \frac{\kappa}{2}\right\}>2 T
$$

and set $t_{2}:=\bar{t}_{2}-s+T<t_{1}$. Consequently,

$$
\lambda\left(\bar{t}_{2}-s, \bar{t}_{2}\right) U_{\kappa}\left(S^{i}\right)=\lambda\left(t_{2}-T, t_{2}\right) \underbrace{\lambda\left(t_{2}, \bar{t}_{2}\right) U_{\kappa}\left(S^{i}\right)}_{\subset U_{\kappa / 2}\left(S^{i}\right)} \stackrel{(5.11)}{\in} U_{\kappa / 4}\left(S^{i}\right)
$$


holds. This is a contradiction, i.e., we have $\lambda\left(t_{2}-t, t_{2}\right) U_{\kappa}\left(S^{i}\right) \subset U_{\kappa / 2}\left(S^{i}\right)$ for all $t_{2}<t_{1}$ and $t>T$. Since $\mu(t) \in U_{\kappa / 6}(0)$ for all $t<t_{1}$, the relation (5.8) is fulfilled.

Step 4. Existence of the past repeller.

Repeated usage of Step 3 implies

$$
\lim _{t \rightarrow-\infty} d\left(\lambda(t, \tau) U_{5 \eta / 6}\left(\mathcal{A}_{\mu}+\mu(\tau)\right) \mid \mathcal{A}_{\mu}+\mu(t)\right)=0 \text { for all } \tau<t_{1} .
$$

Because of Theorem $4.3(\mathrm{i})$, there exist an $s<\tau$ and a past repeller $R \subset(-\infty, s) \times D$ with int $\mathcal{A}_{\mu} \subset \liminf _{t \rightarrow-\infty}(R(t)-\mu(t)) \subset \lim \sup _{t \rightarrow-\infty}(R(t)-\mu(t)) \subset \operatorname{cls} \mathcal{A}_{\mu}$. Since $\lim _{t \rightarrow-\infty} \mu(t)=0$, we have $S^{i} \subset \liminf _{t \rightarrow-\infty} R(t) \subset \limsup _{t \rightarrow-\infty} R(t) \subset \operatorname{cls} S^{i}$. This finishes the proof of this lemma.

The proof of (ii) is similar to that of (i), but there are some differences; for instance, one has to use Theorem 4.3 (ii) instead of Theorem 4.3 (i) in Step 4, and the assumptions of these two theorems are quite different (see [26, Lemma 7.1.6] for the complete proof).

\section{Bifurcations in Dimension One}

In this section, one-dimensional differential equations are studied which exhibit pitchfork, transcritical or saddle node bifurcations. It is shown that, under special assumptions, the bifurcation behavior is transferred to asymptotically autonomous systems.

Let $-\infty \leq x_{-}<x_{+} \leq \infty$ and $\alpha_{0}<\alpha_{1}$, and consider an autonomous differential equation

$$
\dot{x}=g(x, \alpha)
$$

depending on a parameter $\alpha$ with a $C^{1}$-function $g:\left(x_{-}, x_{+}\right) \times\left(\alpha_{0}, \alpha_{1}\right] \rightarrow \mathbb{R}$. We assume that there exists an $x_{0} \in\left(x_{-}, x_{+}\right)$with

$$
g\left(x_{0}, \alpha\right)=0 \quad \text { and } \quad D_{1} g\left(x_{0}, \alpha\right) \neq 0 \quad \text { for all } \alpha \in\left(\alpha_{0}, \alpha_{1}\right] .
$$

Moreover, we consider the nonautonomous differential equation

$$
\dot{x}=f(t, x, \alpha)
$$

depending on a parameter $\alpha$ with a $C^{1}$-function $f:(-\infty, 0) \times\left(x_{-}, x_{+}\right) \times\left(\alpha_{0}, \alpha_{1}\right] \rightarrow$ $\mathbb{R}$ and assume that

$$
\lim _{t \rightarrow-\infty} f(t, x, \alpha)=g(x, \alpha) \quad \text { and } \quad \lim _{t \rightarrow-\infty} D_{2} f(t, x, \alpha)=D_{1} g(x, \alpha)
$$

hold uniformly for all $x \in\left(x_{-}, x_{+}\right)$and $\alpha \in\left(\alpha_{0}, \alpha_{1}\right]$. We denote the general solution of $(6.2)$ by $\lambda$.

In the following, we study solutions of (6.2) which are nonautonomous counterparts for the equilibrium $x_{0}$ of (6.1). In a first instance, the parameter area is restricted to compact subintervals of $\left(\alpha_{0}, \alpha_{1}\right]$, and furthermore, the equilibrium $x_{0}$ is supposed to be attractive.

The statement (i) of the following lemma corresponds to the autonomous pitchfork bifurcation, where after the bifurcation, there are three equilibria, and (ii) and (iii) describe the situation after a transcritical or saddle node bifurcation.

Lemma 6.1. Let $\alpha_{-} \leq \alpha_{+}$be in $\left(\alpha_{0}, \alpha_{1}\right]$, and suppose that

$$
D_{1} g\left(x_{0}, \alpha\right)<0 \text { for all } \alpha \in\left[\alpha_{-}, \alpha_{+}\right] \text {. }
$$

Then there exist a $\tau<0$ and a continuous function $\mu:(-\infty, \tau] \times\left[\alpha_{-}, \alpha_{+}\right] \rightarrow \mathbb{R}$ such that $\mu(\cdot, \alpha)$ is the uniquely determined past attractive solution of $(6.2)$ which 
fulfills $\lim _{t \rightarrow-\infty} \mu(t, \alpha)=x_{0}$. In addition, for fixed $\alpha \in\left[\alpha_{-}, \alpha_{+}\right]$, the following statements are fulfilled:

(i) If there exist $x_{0}^{-}<x_{0}$ and $x_{0}^{+}>x_{0}$ with

$$
\begin{gathered}
g\left(x_{0}^{-}, \alpha\right)=g\left(x_{0}^{+}, \alpha\right)=0, \quad D_{1} g\left(x_{0}^{-}, \alpha\right)>0 \quad \text { and } D_{1} g\left(x_{0}^{+}, \alpha\right)>0, \\
\text { and } g(x, \alpha) \neq 0 \text { for all } x \in\left(x_{0}^{-}, x_{0}\right) \cup\left(x_{0}, x_{0}^{+}\right) \text {, we have } \\
\operatorname{int} \mathcal{A}_{\mu(\cdot, \alpha)}=\left(x_{0}^{-}-x_{0}, x_{0}^{+}-x_{0}\right) .
\end{gathered}
$$

Furthermore, there exists a past repeller $R(\alpha)$ of (6.2) with

$$
\left(x_{0}^{-}, x_{0}^{+}\right) \subset \liminf _{t \rightarrow-\infty} R(\alpha, t) \subset \limsup _{t \rightarrow-\infty} R(\alpha, t) \subset\left[x_{0}^{-}, x_{0}^{+}\right] .
$$

(ii) If there exists a $x_{0}^{-}<x_{0}$ with

$$
g\left(x_{0}^{-}, \alpha\right)=0 \quad \text { and } \quad D_{1} g\left(x_{0}^{-}, \alpha\right)>0,
$$

and $g(x, \alpha) \neq 0$ for all $x \in\left(x_{0}^{-}, x_{0}\right) \cup\left(x_{0}, x_{+}\right)$, we have

$$
\operatorname{int} \mathcal{A}_{\mu(\cdot, \alpha)}=\left(x_{0}^{-}-x_{0}, x_{+}-x_{0}\right) \text {. }
$$

(iii) If there exists $a x_{0}^{+}>x_{0}$ with

$$
\begin{gathered}
\qquad\left(x_{0}^{+}, \alpha\right)=0 \text { and } D_{1} g\left(x_{0}^{+}, \alpha\right)>0, \\
\text { and } g(x, \alpha) \neq 0 \text { for all } x \in\left(x_{-}, x_{0}\right) \cup\left(x_{0}, x_{0}^{+}\right) \text {, we have } \\
\operatorname{int} \mathcal{A}_{\mu(\cdot, \alpha)}=\left(x_{-}-x_{0}, x_{0}^{+}-x_{0}\right) .
\end{gathered}
$$

Proof. The proof is divided into three steps.

Step 1. There exist a $\tau<0$ and a continuous function $\mu:(-\infty, \tau] \times\left[\alpha_{-}, \alpha_{+}\right] \rightarrow \mathbb{R}$ such that $\mu(\cdot, \alpha)$ is the uniquely determined past attractive solution of (6.2) which fulfills $\lim _{t \rightarrow-\infty} \mu(t, \alpha)=x_{0}$.

Due to the hypotheses (please note that $\left[\alpha_{-}, \alpha_{+}\right]$is compact and $g$ is uniformly continuous on compact sets), there exist $\beta>0, \gamma<0$ and $\tau<0$ with

$$
f\left(t, x_{0}-\beta, \alpha\right)>0, f\left(t, x_{0}+\beta, \alpha\right)<0 \quad \text { and } \quad D_{2} f(t, x, \alpha) \leq \gamma
$$

for all $x \in U_{2 \beta}\left(x_{0}\right), t \leq \tau$ and $\alpha \in\left[\alpha_{-}, \alpha_{+}\right]$. We fix an $\alpha \in\left[\alpha_{-}, \alpha_{+}\right]$for the rest of this step. The sets

$$
M_{1}:=\left\{x \in \operatorname{cls} U_{\beta}\left(x_{0}\right): \text { There exists a } t<\tau \text { with } \lambda(t, \tau, \alpha) x<x_{0}-\beta\right\}
$$

and $\quad M_{2}:=\left\{x \in \operatorname{cls} U_{\beta}\left(x_{0}\right):\right.$ There exists a $t<\tau$ with $\left.\lambda(t, \tau, \alpha) x>x_{0}+\beta\right\}$

are obviously nonempty and due to the continuity of $\lambda$ relatively open in $\operatorname{cls} U_{\beta}\left(x_{0}\right)$. Hence, $M_{1} \cup M_{2} \subsetneq \operatorname{cls} U_{\beta}\left(x_{0}\right)$, and therefore, there exists a $y \in U_{\beta}\left(x_{0}\right)$ such that $\mu(t, \alpha):=\lambda(t, \tau, \alpha) y \in U_{\beta}\left(x_{0}\right)$ for all $t \leq \tau$. To show that this solution is past attractive, we study the differential equation of the perturbed motion

$$
\dot{x}=h(t, x, \alpha):=f(t, x+\mu(t, \alpha), \alpha)-f(t, \mu(t, \alpha), \alpha),
$$

whose general solution will be denoted by $\tilde{\lambda}$. The mean value theorem implies $h(t, x, \alpha)=x \int_{0}^{1} D_{2} h(t, \theta x, \alpha) d \theta=x \int_{0}^{1} D_{2} f(t, \theta x+\mu(t, \alpha), \alpha) d \theta$, and thus,

$$
h(t, x, \alpha) \geq \gamma x \text { for all } t \leq \tau \text { and } x \in(-\beta, 0)
$$$$
\text { and } \quad h(t, x, \alpha) \leq \gamma x \text { for all } t \leq \tau \text { and } x \in(0, \beta) .
$$

holds. We obtain $\lim _{t \rightarrow-\infty} d\left(\tilde{\lambda}(\tau, t, \alpha) U_{\beta / 2}(0) \mid\{0\}\right)=0$, and consequently,

$$
\lim _{t \rightarrow-\infty} d\left(\lambda(\tau, t, \alpha) U_{\frac{\beta}{2}}(\mu(t, \alpha)) \mid\{\mu(\tau, \alpha)\}\right)=0
$$


holds. Thus, the solution $\mu(\cdot, \alpha)$ is past attractive. Moreover, the limit relation $\lim _{t \rightarrow-\infty} \mu(t, \alpha)=x_{0}$ is obviously fulfilled. To show the uniqueness of $\mu(\cdot, \alpha)$, we suppose the existence of a value $z \in U_{\beta}\left(x_{0}\right), y \neq z$, with $\nu(t):=\lambda(t, \tau, \alpha) z \in U_{\beta}\left(x_{0}\right)$ for all $t \leq \tau$. Since there exists a $\tilde{t} \leq \tau$ such that $\nu(t) \in U_{\beta / 2}(\mu(t, \alpha))$ for $t \leq \tilde{t}$, we have

$$
0=\lim _{t \rightarrow-\infty}(\lambda(\tau, t, \alpha) \nu(t)-\mu(\tau))=\nu(\tau)-\mu(\tau) \neq 0 .
$$

This is a contradiction, which means that the solution $\mu$ is uniquely determined.

Step 2. $\mu$ is continuous.

First, we consider the function $d:\left[\alpha_{-}, \alpha_{+}\right] \rightarrow\left(x_{-}, x_{+}\right)$, defined by $d(\alpha):=$ $\mu(\tau, \alpha)$ for all $\alpha \in\left[\alpha_{-}, \alpha_{+}\right]$. Suppose, there exist a $\tilde{\alpha} \in\left[\alpha_{-}, \alpha_{+}\right]$and a sequence $\left\{\tilde{\alpha}_{n}\right\}_{n \in \mathbb{N}}$ with $\lim _{n \rightarrow \infty} \tilde{\alpha}_{n}=\tilde{\alpha}$ such that $\left\{d\left(\tilde{\alpha}_{n}\right)\right\}_{n \in \mathbb{N}}$ does not converge to $d(\tilde{\alpha})$. Since this sequence is bounded, we assume w.l.o.g. that it is convergent with limit $\tilde{x} \in \operatorname{cls} U_{\beta}\left(x_{0}\right), \tilde{x} \neq d(\tilde{\alpha})$. Due to Step 1 , there exists a $\tilde{t}<\tau$ with $\lambda(\tilde{t}, \tau, \tilde{\alpha}) \tilde{x} \notin \operatorname{cls} U_{\beta}\left(x_{0}\right)$. The continuity of the general solution implies the existence of a neighborhood $V$ of $(\tilde{x}, \tilde{\alpha})$ such that $\lambda(\tilde{t}, \tau, \alpha) x \notin \operatorname{cls} U_{\beta}\left(x_{0}\right)$ for all $(x, \alpha) \in V$. In particular, there exists an $n \in \mathbb{N}$ with $\left(d\left(\tilde{\alpha}_{n}\right), \tilde{\alpha}_{n}\right) \in V$. This implies

$$
\mu\left(\tilde{t}, \tilde{\alpha}_{n}\right)=\lambda\left(\tilde{t}, \tau, \tilde{\alpha}_{n}\right) \mu\left(\tau, \tilde{\alpha}_{n}\right)=\lambda\left(\tilde{t}, \tau, \tilde{\alpha}_{n}\right) d\left(\tilde{\alpha}_{n}\right) \notin \operatorname{cls} U_{\beta}\left(x_{0}\right) .
$$

This is a contradiction, and therefore, the function $d$ is continuous. To prove the continuity of $\mu$, we choose a sequence $\left\{\left(\hat{t}_{n}, \hat{\alpha}_{n}\right)\right\}_{n \in \mathbb{N}}$ in $(-\infty, \tau] \times\left[\alpha_{-}, \alpha_{+}\right]$with $\lim _{n \rightarrow \infty}\left(\hat{t}_{n}, \hat{\alpha}_{n}\right)=(\hat{t}, \hat{\alpha})$. The continuity of $\mu$ follows from

$$
\begin{aligned}
\lim _{n \rightarrow \infty} \mu\left(\hat{t}_{n}, \hat{\alpha}_{n}\right) & =\lim _{n \rightarrow \infty} \lambda\left(\hat{t}_{n}, \tau, \hat{\alpha}_{n}\right) \mu\left(\tau, \hat{\alpha}_{n}\right)=\lim _{n \rightarrow \infty} \lambda\left(\hat{t}_{n}, \tau, \hat{\alpha}_{n}\right) d\left(\hat{\alpha}_{n}\right) \\
& =\lambda(\hat{t}, \tau, \hat{\alpha}) d(\hat{\alpha})=\mu(\hat{t}, \hat{\alpha}) .
\end{aligned}
$$

Step 3. The statements (i), (ii) and (iii) are fulfilled.

The asserted relations for $\mathcal{A}_{\mu(\cdot, \alpha)}$ and the existence of a past repeller follow directly from Lemma 5.4 if we define the repulsive set $S$ as $\left\{x_{0}^{-}, x_{0}^{+}\right\}$in case (i), $\left\{x_{0}^{-}\right\}$in case (ii) or $\left\{x_{0}^{+}\right\}$in case (iii), respectively (cf. also Figure 1).

Under the assumption $D_{1} g\left(x_{0}, \alpha\right)>0$ for all $\alpha \in\left[\alpha_{-}, \alpha_{+}\right]$, analogous statements are obtained for past repulsive solutions.

Lemma 6.2. Let $\alpha_{-} \leq \alpha_{+}$be in $\left(\alpha_{0}, \alpha_{1}\right]$, and suppose that

$$
D_{1} g\left(x_{0}, \alpha\right)>0 \text { for all } \alpha \in\left[\alpha_{-}, \alpha_{+}\right] \text {. }
$$

Then there exist $\tau<0$ and $\beta>0$ such that every solution $\lambda(\cdot, \tau, \alpha) x$ for $x \in U_{\beta}\left(x_{0}\right)$ and $\alpha \in\left[\alpha_{-}, \alpha_{+}\right]$is past repulsive with $\lim _{t \rightarrow-\infty} \lambda(t, \tau, \alpha) x=x_{0}$. Let $\nu:(-\infty, \tau] \rightarrow$ $\mathbb{R}$ be such a solution of (6.2) for fixed $\alpha \in\left[\alpha_{-}, \alpha_{+}\right]$. Then the following statements are fulfilled:

(i) If there exist $x_{0}^{-}<x_{0}$ and $x_{0}^{+}>x_{0}$ with

$$
\begin{gathered}
g\left(x_{0}^{-}, \alpha\right)=g\left(x_{0}^{+}, \alpha\right)=0, \quad D_{1} g\left(x_{0}^{-}, \alpha\right)<0 \quad \text { and } D_{1} g\left(x_{0}^{+}, \alpha\right)<0, \\
\text { and } g(x, \alpha) \neq 0 \text { for all } x \in\left(x_{0}^{-}, x_{0}\right) \cup\left(x_{0}, x_{0}^{+}\right) \text {, we have } \\
\operatorname{int} \mathcal{R}_{\nu}=\left(x_{0}^{-}-x_{0}, x_{0}^{+}-x_{0}\right) .
\end{gathered}
$$

Furthermore, there exists a past attractor $A(\alpha)$ of (6.2) with

$$
\left(x_{0}^{-}, x_{0}^{+}\right) \subset \liminf _{t \rightarrow-\infty} A(\alpha, t) \subset \limsup _{t \rightarrow-\infty} A(\alpha, t) \subset\left[x_{0}^{-}, x_{0}^{+}\right] .
$$


(ii) If there exists a $x_{0}^{-}<x_{0}$ with

$$
g\left(x_{0}^{-}, \alpha\right)=0 \quad \text { and } \quad D_{1} g\left(x_{0}^{-}, \alpha\right)<0,
$$

and $g(x, \alpha) \neq 0$ for all $x \in\left(x_{0}^{-}, x_{0}\right) \cup\left(x_{0}, x_{+}\right)$, we have

$$
\operatorname{int} \mathcal{R}_{\nu}=\left(x_{0}^{-}-x_{0}, x_{+}-x_{0}\right) \text {. }
$$

(iii) If there exists a $x_{0}^{+}>x_{0}$ with

$$
\begin{gathered}
\qquad\left(x_{0}^{+}, \alpha\right)=0 \quad \text { and } D_{1} g\left(x_{0}^{+}, \alpha\right)<0, \\
\text { and } g(x, \alpha) \neq 0 \text { for all } x \in\left(x_{-}, x_{0}\right) \cup\left(x_{0}, x_{0}^{+}\right) \text {, we have } \\
\operatorname{int} \mathcal{R}_{\nu}=\left(x_{-}-x_{0}, x_{0}^{+}-x_{0}\right) .
\end{gathered}
$$

Proof. Due to the hypotheses (please note that $\left[\alpha_{-}, \alpha_{+}\right]$is compact and $g$ is uniformly continuous on compact sets), there exist $\beta>0, \gamma>0$ and $\tau<0$ with

$$
f\left(t, x_{0}-\beta, \alpha\right)<0, f\left(t, x_{0}+\beta, \alpha\right)>0 \quad \text { and } \quad D_{2} f(t, x, \alpha) \geq \gamma
$$

for all $x \in U_{2 \beta}\left(x_{0}\right), t \leq \tau$ and $\alpha \in\left[\alpha_{-}, \alpha_{+}\right]$. We fix $\hat{x} \in U_{\beta}\left(x_{0}\right)$ and $\alpha \in\left[\alpha_{-}, \alpha_{+}\right]$ and consider for the rest of the proof in particular the solution $\nu(\cdot):=\lambda(\cdot, \tau, \alpha) \hat{x}$ of $(6.2)$ on the interval $(-\infty, \tau]$. Obviously, the relation $\lim _{t \rightarrow-\infty} \nu(t)=x_{0}$ holds. To show that this solution is past repulsive, we study the differential equation of the perturbed motion $\dot{x}=h(t, x, \alpha):=f(t, x+\nu(t), \alpha)-f(t, \nu(t), \alpha)$, whose general solution will be denoted by $\tilde{\lambda}$. Due to the mean value theorem, we have $h(t, x, \alpha)=x \int_{0}^{1} D_{2} h(t, \theta x, \alpha) d \theta=x \int_{0}^{1} D_{2} f(t, \theta x+\nu(t), \alpha) d \theta$. This implies

$$
\begin{aligned}
h(t, x, \alpha) \leq \gamma x & \text { for all } t \leq \tau \text { and } x \in(-\beta, 0) \\
\text { and } \quad h(t, x, \alpha) \geq \gamma x & \text { for all } t \leq \tau \text { and } x \in(0, \beta) .
\end{aligned}
$$

We obtain $\lim _{t \rightarrow-\infty} d\left(\tilde{\lambda}(t, s, \alpha) U_{\beta / 2}(0) \mid\{0\}\right)=0$ for all $s \leq \tau$, and consequently,

$$
\lim _{t \rightarrow-\infty} d\left(\lambda(t, s, \alpha) U_{\frac{\beta}{2}}(\nu(s)) \mid\{\nu(t)\}\right)=0 \quad \text { for all } s \leq \tau
$$

holds. Thus, the solution $\nu$ is past repulsive. The asserted relations for $\mathcal{R}_{\nu}$ and the existence of a past attractor follow directly from Lemma 5.4 if we define the repulsive set $S$ as $\left\{x_{0}^{-}, x_{0}^{+}\right\}$in case (i), $\left\{x_{0}^{-}\right\}$in case (ii) or $\left\{x_{0}^{+}\right\}$in case (iii), respectively (cf. also Figure 2).

In the following, we observe that pitchfork bifurcations of (6.1) give rise to total past bifurcations. Transcritical and saddle node bifurcations, however, lead to partial past bifurcations.

First, the attention is restricted to the situation that the autonomous differential equation (6.1) admits a supercritical pitchfork bifurcation at $\left(x_{0}, \alpha_{0}\right)$. More precisely, there exist a monotone increasing continuous function $h_{1}:\left(\alpha_{0}, \alpha_{1}\right] \rightarrow$ $\left(x_{-}, x_{+}\right)$and a monotone decreasing continuous function $h_{2}:\left(\alpha_{0}, \alpha_{1}\right] \rightarrow\left(x_{-}, x_{+}\right)$ such that for all $\alpha \in\left(\alpha_{0}, \alpha_{1}\right]$, we have

$$
\begin{gathered}
h_{1}(\alpha)<x_{0}<h_{2}(\alpha), \quad g\left(h_{1}(\alpha), \alpha\right)=g\left(x_{0}, \alpha\right)=g\left(h_{2}(\alpha), \alpha\right)=0, \\
D_{1} g\left(h_{1}(\alpha), \alpha\right) \neq 0, D_{1} g\left(x_{0}, \alpha\right) \neq 0, D_{1} g\left(h_{2}(\alpha), \alpha\right) \neq 0 .
\end{gathered}
$$

Moreover, for all $\alpha \in\left(\alpha_{0}, \alpha_{1}\right]$ and $x \in\left(h_{1}(\alpha), x_{0}\right) \cup\left(x_{0}, h_{2}(\alpha)\right), g(x, \alpha) \neq 0$ is satisfied, and we have $\lim _{\alpha \rightarrow \alpha_{0}} h_{1}(\alpha)=\lim _{\alpha \rightarrow \alpha_{0}} h_{2}(\alpha)=x_{0}$.

Theorem 6.3. The following statements are fulfilled: 
(i) If $D_{1} g\left(x_{0}, \alpha_{1}\right)<0$, then there exists a continuous function $\mu: D \subset \mathbb{R} \times$ $\left(\alpha_{0}, \alpha_{1}\right] \rightarrow \mathbb{R}$ such that $\mu(\cdot, \alpha)$ is a past attractive solution of $(6.2)$. We have a total past bifurcation, since

$$
\lim _{\alpha \searrow \alpha_{0}} d\left(\mathcal{A}_{\mu(\cdot, \alpha)} \mid\{0\}\right)=0 .
$$

Furthermore, for all $\alpha \in\left(\alpha_{0}, \alpha_{1}\right]$, there exists a past repeller $R(\alpha)$. Due to

$$
\lim _{\alpha \searrow \alpha_{0}} d\left(\limsup _{t \rightarrow-\infty} R(\alpha, t) \mid\left\{x_{0}\right\}\right)=0,
$$

we also have a past repeller transition.

(ii) If $D_{1} g\left(x_{0}, \alpha_{1}\right)>0$, then there exists a continuous function $\mu: D \subset \mathbb{R} \times$ $\left(\alpha_{0}, \alpha_{1}\right] \rightarrow \mathbb{R}$ such that $\mu(\cdot, \alpha)$ is a past repulsive solution of $(6.2)$. We have a total past bifurcation, since

$$
\lim _{\alpha \searrow \alpha_{0}} d\left(\mathcal{R}_{\mu(\cdot, \alpha)} \mid\{0\}\right)=0 .
$$

Furthermore, for all $\alpha \in\left(\alpha_{0}, \alpha_{1}\right]$, there exists a past attractor $A(\alpha)$. Due to

$$
\lim _{\alpha \searrow \alpha_{0}} d\left(\limsup _{t \rightarrow-\infty} A(\alpha, t) \mid\left\{x_{0}\right\}\right)=0,
$$

we also have a past attractor transition.

Proof. We define the compact intervals $I_{0}:=\left\{\alpha \in\left(\alpha_{0}, \alpha_{1}\right]: h_{1}(\alpha) \leq x_{0}-1\right\}$ and $I_{n}:=\left\{\alpha \in\left(\alpha_{0}, \alpha_{1}\right]: h_{1}(\alpha) \in\left[x_{0}-1 / n, x_{0}-1 /(n+1)\right]\right\}$ for all $n \in \mathbb{N}$.

(i) For $n \in \mathbb{N}_{0}$, we restrict (6.2) to the parameter area $I_{n}$ and apply Lemma 6.1. Therefore, there exists a continuous function $\mu_{n}:\left(-\infty, \tau_{n}\right] \times I_{n} \rightarrow \mathbb{R}$ which describes uniquely determined past attractive solutions. We define $\mu(t, \alpha):=\mu_{n}(t, \alpha)$ for all $t<0$ and $\alpha \in\left(\alpha_{0}, \alpha_{1}\right]$ with $\alpha \in I_{n}$ and $t \leq \tau_{n}$. Due to the uniqueness of the $\mu_{n}$, the function $\mu: D \rightarrow \mathbb{R}$ for some $D \subset \mathbb{R} \times\left(\alpha_{0}, \alpha_{1}\right]$ is well defined, and the continuity of $\mu$ follows directly. The existence of the past repellers and the limit relations are consequences of Lemma 6.1 (i).

(ii) For $n \in \mathbb{N}_{0}$, we restrict (6.2) to the parameter area $I_{n}$ and apply Lemma 6.2 . It is obvious that one can construct a continuous function $\mu: D \subset \mathbb{R} \times\left(\alpha_{0}, \alpha_{1}\right) \rightarrow \mathbb{R}$ which describes past repulsive solutions. The existence of the past attractors and the limit relations are consequences of Lemma 6.2 (i).

To obtain partial nonautonomous bifurcations, we assume that the differential equation (6.2) admits a supercritical transcritical or saddle node bifurcation at $\left(x_{0}, \alpha_{0}\right)$. This means, there exists a strictly increasing continuous function $h$ : $\left(\alpha_{0}, \alpha_{1}\right) \rightarrow\left(x_{-}, x_{+}\right)$such that for all $\alpha \in\left(\alpha_{0}, \alpha_{1}\right)$, we have

$$
h(\alpha)<x_{0}, \quad g(h(\alpha), \alpha)=g\left(x_{0}, \alpha\right)=0, \quad D_{1} g(h(\alpha), \alpha) \neq 0, D_{1} g\left(x_{0}, \alpha\right) \neq 0 .
$$

Moreover, for all $\alpha \in\left(\alpha_{0}, \alpha_{1}\right)$ and $x \in\left(h(\alpha), x_{0}\right)$, the relation $g(x, \alpha) \neq 0$ is satisfied, and we have $\lim _{\alpha \rightarrow \alpha_{0}} h(\alpha)=x_{0}$. Please note that in case of a saddle node bifurcation, one has to transform the greater equilibrium into $x_{0}$. In case of a transcritical bifurcation, we assume that the bigger equilibrium equals $x_{0}$. This can be also reached by a transformation.

Theorem 6.4. The following statements are fulfilled: 
(i) If $D_{1} g\left(x_{0}, \alpha_{1}\right)<0$, then there exists a continuous function $\mu: D \subset \mathbb{R} \times$ $\left(\alpha_{0}, \alpha_{1}\right] \rightarrow \mathbb{R}$ such that $\mu(\cdot, \alpha)$ is a past attractive solution of $(6.2)$. We have a partial bifurcation with $\lim _{\alpha \backslash \alpha_{0}} \sup \left\{\gamma>0: U_{\gamma}(0) \subset \mathcal{A}_{\mu(\cdot, \alpha)}\right\}=0$.

(ii) If $D_{1} g\left(x_{0}, \alpha_{1}\right)>0$, then there exists a continuous function $\mu: D \subset \mathbb{R} \times$ $\left(\alpha_{0}, \alpha_{1}\right] \rightarrow \mathbb{R}$ such that $\mu(\cdot, \alpha)$ is a past repulsive solution of $(6.2)$. We have a partial bifurcation with $\lim _{\alpha \searrow \alpha_{0}} \sup \left\{\gamma>0: U_{\gamma}(0) \subset \mathcal{R}_{\mu(\cdot, \alpha)}\right\}=0$.

Proof. See proof of Theorem 6.3.

\section{Bifurcations in Dimension Two}

In this section, two-dimensional differential equations which exhibit Hopf bifurcations are studied (see, e.g., [21]). As in the previous section, this bifurcation behavior is transferred to asymptotically autonomous systems.

More precisely, we consider the autonomous differential equation

$$
\dot{x}=g_{1}(x, y, \alpha), \quad \dot{y}=g_{2}(x, y, \alpha)
$$

depending on a parameter $\alpha$ with a $C^{1}$-function $g:\left(x_{-}, x_{+}\right) \times\left(y_{-}, y_{+}\right) \times\left(\alpha_{0}, \alpha_{1}\right] \rightarrow$ $\mathbb{R}^{2}$ which admits a supercritical Hopf bifurcation at $\left(x_{0}, y_{0}, \alpha_{0}\right)$, i.e., for all $\alpha \in$ $\left(\alpha_{0}, \alpha_{1}\right]$, we have

$$
g\left(x_{0}, y_{0}, \alpha\right)=0 \quad \text { and } \quad D_{(1,2)} g\left(x_{0}, y_{0}, \alpha\right)=\left(\begin{array}{cc}
a(\alpha) & -b(\alpha) \\
b(\alpha) & a(\alpha)
\end{array}\right)
$$

with continuous functions $a:\left(\alpha_{0}, \alpha_{1}\right] \rightarrow \mathbb{R}$ and $b:\left(\alpha_{0}, \alpha_{1}\right] \rightarrow \mathbb{R}$ which fulfill $a(\alpha) \neq 0$ and $b(\alpha) \neq 0$. Furthermore, let $S(\alpha)$ be an attractive (in case $a(\alpha)<0$ ) or a repulsive (in case $a(\alpha)>0$ ) periodic orbit of (7.1), respectively, which depends continuously on $\alpha$ with respect to the Hausdorff distance and converges to $\left(x_{0}, y_{0}\right)$ in the limit $\alpha \rightarrow \alpha_{0}$. We denote the inner area of $S(\alpha)$ by $S^{i}(\alpha)$. Moreover, we consider the nonautonomous differential equation

$$
\dot{x}=f_{1}(t, x, y, \alpha), \quad \dot{y}=f_{2}(t, x, y, \alpha)
$$

depending on a parameter $\alpha$ with a $C^{1}$-function $f:(-\infty, 0) \times\left(x_{-}, x_{+}\right) \times\left(y_{-}, y_{+}\right) \times$ $\left(\alpha_{0}, \alpha_{1}\right] \rightarrow \mathbb{R}^{2}$. We assume that

$$
\lim _{t \rightarrow-\infty} f(t, x, y, \alpha)=g(x, y, \alpha) \quad \text { and } \lim _{t \rightarrow-\infty} D_{(2,3)} f(t, x, y, \alpha)=D_{(1,2)} g(x, y, \alpha)
$$

hold uniformly for all $x \in\left(x_{-}, x_{+}\right), y \in\left(y_{-}, y_{+}\right)$and $\alpha \in\left(\alpha_{0}, \alpha_{1}\right]$. We denote the general solution of $(7.2)$ by $\lambda$.

As in the previous section, we study solutions of (7.2) which are nonautonomous counterparts for the equilibrium $\left(x_{0}, y_{0}\right)$ of $(7.1)$. In a first instance, the parameter area is restricted to compact subintervals of $\left(\alpha_{0}, \alpha_{1}\right]$, and furthermore, the equilibrium $\left(x_{0}, y_{0}\right)$ is supposed to be attractive.

Lemma 7.1. Let $\alpha_{-} \leq \alpha_{+}$be in $\left(\alpha_{0}, \alpha_{1}\right]$, and suppose that

$$
a(\alpha)<0 \quad \text { for all } \alpha \in\left[\alpha_{-}, \alpha_{+}\right] \text {. }
$$

Then there exist a $\tau<0$ and a continuous function $\mu:(-\infty, \tau] \times\left[\alpha_{-}, \alpha_{+}\right] \rightarrow \mathbb{R}^{2}$ such that $\mu(\cdot, \alpha)$ is the uniquely determined past attractive solution of $(7.2)$ which fulfills $\lim _{t \rightarrow-\infty} \mu(t, \alpha)=\left(x_{0}, y_{0}\right)$. Moreover, we have

$$
\operatorname{int} \mathcal{A}_{\mu(\cdot, \alpha)}=S^{i}(\alpha)-\left(x_{0}, y_{0}\right) \quad \text { for all } \alpha \in\left[\alpha_{-}, \alpha_{+}\right] \text {. }
$$


Furthermore, there exists a past repeller $R(\alpha)$ of (7.2) with

$$
S^{i}(\alpha) \subset \liminf _{t \rightarrow-\infty} R(\alpha, t) \subset \limsup _{t \rightarrow-\infty} R(\alpha, t) \subset \operatorname{cls} S^{i}(\alpha) \quad \text { for all } \alpha \in\left[\alpha_{-}, \alpha_{+}\right] .
$$

Proof. W.l.o.g., let $\left(x_{0}, y_{0}\right)=(0,0)$. The proof is divided into three steps.

Step 1. There exists a $\tau<0$ and a continuous function $\mu:(-\infty, \tau] \times\left[\alpha_{-}, \alpha_{+}\right] \rightarrow$ $\mathbb{R}^{2}$ such that $\mu(\cdot, \alpha)$ is the uniquely determined past attractive solution of $(7.2)$ which fulfills $\lim _{t \rightarrow-\infty} \mu(t, \alpha)=(0,0)$.

Due to the compactness of $\left[\alpha_{-}, \alpha_{+}\right]$and the uniform continuity of $g$ on compact sets, there exist $\beta>0$ and $\gamma<0$ with

$$
\frac{\partial g_{1}}{\partial x}(x, y, \alpha) \leq 2 \gamma, \frac{\partial g_{2}}{\partial y}(x, y, \alpha) \leq 2 \gamma \text { and }\left|\frac{\partial g_{1}}{\partial y}(x, y, \alpha)+\frac{\partial g_{2}}{\partial x}(x, y, \alpha)\right| \leq-\gamma
$$

for all $(x, y) \in \operatorname{cls} U_{2 \beta}((0,0))$ and $\alpha \in\left[\alpha_{-}, \alpha_{+}\right]$. This implies the existence of a $\tau<0$ with

$$
\frac{\partial f_{1}}{\partial x}(t, x, y, \alpha) \leq \gamma, \frac{\partial f_{2}}{\partial y}(t, x, y, \alpha) \leq \gamma,\left|\frac{\partial f_{1}}{\partial y}(t, x, y, \alpha)+\frac{\partial f_{2}}{\partial x}(t, x, y, \alpha)\right| \leq-\frac{\gamma}{2}
$$

and $\left|f_{1}(t, 0,0, \alpha)\right|+\left|f_{2}(t, 0,0, \alpha)\right| \leq-\gamma \beta / 4$ for all $t \leq \tau, x, y \in \operatorname{cls} U_{2 \beta}((0,0))$ and $\alpha \in\left[\alpha_{-}, \alpha_{+}\right]$. For the rest of this step, we fix an $\alpha \in\left[\alpha_{-}, \alpha_{+}\right]$. The mean value theorem implies $f(t, x, y, \alpha)=f(t, 0,0, \alpha)+\int_{0}^{1} D_{(2,3)} f(t, \theta x, \theta y, \alpha) \cdot(x, y) d \theta$ for all $t \leq \tau$ and $x, y \in \operatorname{cls} U_{2 \beta}((0,0))$. It follows that for all $t \leq \tau, \alpha \in\left[\alpha_{-}, \alpha_{+}\right]$and $x, y$ with $x^{2}+y^{2}=\beta^{2}$,

$$
\begin{aligned}
& \langle f(t, x, y, \alpha),(x, y)\rangle=f_{1}(t, x, y, \alpha) x+f_{2}(t, x, y, \alpha) y \\
= & f_{1}(t, 0,0, \alpha) x+f_{2}(t, 0,0, \alpha) y+\int_{0}^{1}\left(\frac{\partial f_{1}}{\partial x}(t, \theta x, \theta y, \alpha) x^{2}+\frac{\partial f_{2}}{\partial y}(t, \theta x, \theta y, \alpha) y^{2}+\right. \\
& \left.\frac{\partial f_{1}}{\partial y}(t, \theta x, \theta y, \alpha) x y+\frac{\partial f_{2}}{\partial x}(t, \theta x, \theta y, \alpha) x y\right) d \theta \\
\leq & -\frac{\gamma \beta^{2}}{4}+\int_{0}^{1}\left(\gamma x^{2}+\gamma y^{2}-\frac{\gamma}{2}|x y|\right) d \theta \leq \frac{\gamma \beta^{2}}{4}<0
\end{aligned}
$$

holds. Thus, the subset $\operatorname{cls} U_{\beta}((0,0))$ of the phase space is forward invariant in the following sense: We have $\lambda\left(t^{+}, t^{-}, \alpha\right) \operatorname{cls} U_{\beta}((0,0)) \subset \operatorname{cls} U_{\beta}((0,0))$ for all $t^{-} \leq t^{+} \leq$ $\tau$ and $\alpha \in\left[\alpha_{-}, \alpha_{+}\right]$. Hence, the set

$M:=\left\{(x, y) \in \operatorname{cls} U_{\beta}((0,0)):\right.$ There exists a $t \leq \tau$ with $\|\lambda(t, \tau, \alpha)(x, y)\|=\beta$,

$$
\lambda(t, \tau, \alpha)(x, y) \neq(\beta, 0) \text { and } \lambda(t, \tau, \alpha)(x, y) \neq(-\beta, 0)\}
$$

is nonempty and due to the continuity of the general solution relatively open in $\operatorname{cls} U_{\beta}((0,0))$. This means that $\tilde{M}:=\operatorname{cls} U_{\beta}((0,0)) \backslash M$ is closed. The sets

$$
\begin{aligned}
M_{1}:=\{(x, y) \in \tilde{M}: & \text { There exists a } t \leq \tau \text { with } \\
& \|\lambda(t, \tau, \alpha)(x, y)\|=\beta \text { and } \lambda(t, \tau, \alpha)(x, y)=(\beta, 0)\}
\end{aligned}
$$

and $\quad M_{2}:=\{(x, y) \in \tilde{M}$ : There exists a $t \leq \tau$ with

$$
\|\lambda(t, \tau, \alpha)(x, y)\|=\beta \text { and } \lambda(t, \tau, \alpha)(x, y)=(-\beta, 0)\}
$$

are obviously nonempty and due to the continuity of the general solution relatively open in $\tilde{M}$. This implies that $M_{1} \cup M_{2} \subsetneq \tilde{M}$. Thus, there exists a $(\hat{x}, \hat{y}) \in U_{\beta}(0,0)$ 
with $\mu(t, \alpha):=\lambda(t, \tau, \alpha)(\hat{x}, \hat{y}) \in U_{\beta}((0,0))$ for all $t \leq \tau$. To show that $\mu$ is past attractive, we study the differential equation of the perturbed motion

$$
\begin{aligned}
& \dot{x}=h_{1}(t, x, y, \alpha):=f_{1}\left(t, x+\mu_{1}(t, \alpha), y+\mu_{2}(t, \alpha), \alpha\right)-f_{1}(t, \mu(t, \alpha), \alpha) \\
& \dot{y}=h_{2}(t, x, y, \alpha):=f_{2}\left(t, x+\mu_{1}(t, \alpha), y+\mu_{2}(t, \alpha), \alpha\right)-f_{2}(t, \mu(t, \alpha), \alpha)
\end{aligned}
$$

Due to the mean value theorem, for all $t \leq \tau$ and $(x, y) \in U_{\beta}((0,0))$,

$$
\begin{aligned}
h(t, x, y, \alpha) & =\int_{0}^{1} D_{(2,3)} h(t, \theta x, \theta y, \alpha) \cdot(x, y) d \theta \\
& =\int_{0}^{1} D_{(2,3)} f\left(t, \theta x+\mu_{1}(t, \alpha), \theta y+\mu_{2}(t, \alpha), \alpha\right) \cdot(x, y) d \theta
\end{aligned}
$$

is fulfilled. Thus, for all $(r, \varphi) \in(0, \beta) \times[0,2 \pi)$ and $t \leq \tau$, we have

$$
\begin{gathered}
h_{1}(t, r \cos \varphi, r \sin \varphi, \alpha) \cos \varphi+h_{2}(t, r \cos \varphi, r \sin \varphi, \alpha) \sin \varphi \\
=\int_{0}^{1}\left(\frac{\partial f_{1}}{\partial x}\left(t, \theta r \cos \varphi+\mu_{1}(t, \alpha), \theta r \sin \varphi+\mu_{2}(t, \alpha), \alpha\right) r \cos ^{2} \varphi+\right. \\
\quad \frac{\partial f_{2}}{\partial y}\left(t, \theta r \cos \varphi+\mu_{1}(t, \alpha), \theta r \sin \varphi+\mu_{2}(t, \alpha), \alpha\right) r \sin ^{2} \varphi+ \\
\frac{\partial f_{1}}{\partial y}\left(t, \theta r \cos \varphi+\mu_{1}(t, \alpha), \theta r \sin \varphi+\mu_{2}(t, \alpha), \alpha\right) r \cos \varphi \sin \varphi+ \\
\left.\quad \frac{\partial f_{2}}{\partial x}\left(t, \theta r \cos \varphi+\mu_{1}(t, \alpha), \theta r \sin \varphi+\mu_{2}(t, \alpha), \alpha\right) r \cos \varphi \sin \varphi\right) d \theta \\
\leq \int_{0}^{1}\left(\gamma r \cos ^{2} \varphi+\gamma r \sin ^{2} \varphi-\frac{\gamma}{2} r \cos \varphi \sin \varphi\right) d \theta \leq r \frac{\gamma}{2} .
\end{gathered}
$$

Applying polar coordinates, we see that $\mu(\cdot, \alpha)$ is past attractive. Moreover, the limit relation $\lim _{t \rightarrow-\infty} \mu(t, \alpha)=(0,0)$ is obviously satisfied. The uniqueness of $\mu(\cdot, \alpha)$ can be proved analogously as in the proof of Lemma 6.1.

Step 2. $\mu$ is continuous.

See Step 2 of the proof of Lemma 6.1.

Step 3. The assertions concerning $\mathcal{A}_{\mu(\cdot, \alpha)}$ and the past repellers are fulfilled.

This follows directly from Lemma 5.4 (i).

Lemma 7.2. Let $\alpha_{-} \leq \alpha_{+}$be in $\left(\alpha_{0}, \alpha_{1}\right]$, and suppose that

$$
a(\alpha)>0 \text { for all } \alpha \in\left[\alpha_{-}, \alpha_{+}\right] .
$$

Then there exist $\tau<0$ and $\beta>0$ such that for all $(x, y) \in U_{\beta}\left(\left(x_{0}, y_{0}\right)\right)$ and each parameter value $\alpha \in\left[\alpha_{-}, \alpha_{+}\right]$, the solution $\lambda(\cdot, \tau, \alpha)(x, y)$ of $(7.2)$ is past repulsive with $\lim _{t \rightarrow-\infty} \lambda(t, \tau, \alpha)(x, y)=\left(x_{0}, y_{0}\right)$ and

$$
\operatorname{int} \mathcal{R}_{\lambda(\cdot, \tau, \alpha)(x, y)}=S^{i}(\alpha)-\left(x_{0}, y_{0}\right) .
$$

Furthermore, there exists a past attractor $A(\alpha)$ of (7.2) with

$$
S^{i}(\alpha) \subset \liminf _{t \rightarrow-\infty} A(\alpha, t) \subset \limsup _{t \rightarrow-\infty} A(\alpha, t) \subset \operatorname{cls} S^{i}(\alpha) \quad \text { for all } \alpha \in\left[\alpha_{-}, \alpha_{+}\right] .
$$

Proof. W.l.o.g., let $\left(x_{0}, y_{0}\right)=(0,0)$. Due to the compactness of $\left[\alpha_{-}, \alpha_{+}\right]$and the uniform continuity of $g$ on compact sets, there exist $\beta>0$ and $\gamma>0$ with

$$
\frac{\partial g_{1}}{\partial x}(x, y, \alpha) \geq 2 \gamma, \frac{\partial g_{2}}{\partial y}(x, y, \alpha) \geq 2 \gamma \text { and }\left|\frac{\partial g_{1}}{\partial y}(x, y, \alpha)+\frac{\partial g_{2}}{\partial x}(x, y, \alpha)\right| \leq \gamma
$$


for all $(x, y) \in \operatorname{cls} U_{2 \beta}((0,0))$ and $\alpha \in\left[\alpha_{-}, \alpha_{+}\right]$. This implies the existence of a $\tau<0$ with

$$
\frac{\partial f_{1}}{\partial x}(t, x, y, \alpha) \geq \gamma, \frac{\partial f_{2}}{\partial y}(t, x, y, \alpha) \geq \gamma,\left|\frac{\partial f_{1}}{\partial y}(t, x, y, \alpha)+\frac{\partial f_{2}}{\partial x}(t, x, y, \alpha)\right| \leq \frac{\gamma}{2}
$$

and $\left|f_{1}(t, 0,0, \alpha)\right|+\left|f_{2}(t, 0,0, \alpha)\right| \leq \gamma \beta / 4$ for all $t \leq \tau, x, y \in \operatorname{cls} U_{2 \beta}((0,0))$ and $\alpha \in\left[\alpha_{-}, \alpha_{+}\right]$. For the rest of this proof, we fix an $\alpha \in\left[\alpha_{-}, \alpha_{+}\right]$. The mean value theorem implies $f(t, x, y, \alpha)=f(t, 0,0, \alpha)+\int_{0}^{1} f_{x}(t, \theta x, \theta y, \alpha) \cdot(x, y) d \theta$ for all $t \leq \tau$ and $x, y \in \operatorname{cls} U_{2 \beta}((0,0))$. Thus, for all $t \leq \tau$ and $x, y$ with $x^{2}+y^{2}=\beta^{2}$, we have

$$
\begin{aligned}
& \langle f(t, x, y, \alpha),(x, y)\rangle=f_{1}(t, x, y, \alpha) x+f_{2}(t, x, y, \alpha) y \\
= & f_{1}(t, 0,0, \alpha) x+f_{2}(t, 0,0, \alpha) y+\int_{0}^{1}\left(\frac{\partial f_{1}}{\partial x}(t, \theta x, \theta y, \alpha) x^{2}+\frac{\partial f_{2}}{\partial y}(t, \theta x, \theta y, \alpha) y^{2}+\right. \\
& \left.\frac{\partial f_{1}}{\partial y}(t, \theta x, \theta y, \alpha) x y+\frac{\partial f_{2}}{\partial x}(t, \theta x, \theta y, \alpha) x y\right) d \theta \\
\geq & -\frac{\gamma \beta^{2}}{4}+\int_{0}^{1}\left(\gamma x^{2}+\gamma y^{2}-\frac{\gamma}{2}|x y|\right) d \theta \geq \frac{\gamma \beta^{2}}{4}>0 .
\end{aligned}
$$

Therefore, the subset $\operatorname{cls} U_{\beta}((0,0))$ of the phase space is backward invariant in the following sense: We have $\lambda\left(t^{-}, t^{+}, \alpha\right) \operatorname{cls} U_{\beta}((0,0)) \subset \operatorname{cls} U_{\beta}((0,0))$ for all $t^{-} \leq t^{+} \leq$ $\tau$. We choose $(\hat{x}, \hat{y}) \in U_{\beta}((0,0))$ arbitrarily and consider for the rest of this proof in particular the solution $\nu(\cdot):=\lambda(\cdot, \tau, \alpha)(\hat{x}, \hat{y})$ on the interval $(-\infty, \tau]$. It is obvious that $\lim _{t \rightarrow-\infty} \nu(t)=(0,0)$ holds. To show that $\nu$ is past repulsive, we study the differential equation of the perturbed motion

$$
\begin{aligned}
& \dot{x}=h_{1}(t, x, y, \alpha):=f_{1}\left(t, x+\nu_{1}(t), y+\nu_{2}(t), \alpha\right)-f_{1}(t, \nu(t), \alpha) \\
& \dot{y}=h_{2}(t, x, y, \alpha):=f_{2}\left(t, x+\nu_{1}(t), y+\nu_{2}(t), \alpha\right)-f_{2}(t, \nu(t), \alpha)
\end{aligned}
$$

Due to the mean value theorem, we have for all $t \leq \tau$ and $(x, y) \in U_{\beta}((0,0))$,

$$
\begin{aligned}
h(t, x, y, \alpha)= & \int_{0}^{1} D_{(2,3)} h(t, \theta x, \theta y, \alpha) \cdot(x, y) d \theta= \\
& \int_{0}^{1} D_{(2,3)} f\left(t, \theta x+\mu_{1}(t, \alpha), \theta y+\mu_{2}(t, \alpha), \alpha\right) \cdot(x, y) d \theta .
\end{aligned}
$$

Thus, for all $(r, \varphi) \in(0, \beta) \times[0,2 \pi)$ and $t \leq \tau$, we have

$$
\begin{gathered}
h_{1}(t, r \cos \varphi, r \sin \varphi, \alpha) \cos \varphi+h_{2}(t, r \cos \varphi, r \sin \varphi, \alpha) \sin \varphi \\
=\int_{0}^{1}\left(\frac{\partial f_{1}}{\partial x}\left(t, \theta r \cos \varphi+\mu_{1}(t, \alpha), \theta r \sin \varphi+\mu_{2}(t, \alpha), \alpha\right) r \cos ^{2} \varphi+\right. \\
\frac{\partial f_{2}}{\partial y}\left(t, \theta r \cos \varphi+\mu_{1}(t, \alpha), \theta r \sin \varphi+\mu_{2}(t, \alpha), \alpha\right) r \sin ^{2} \varphi+ \\
\frac{\partial f_{1}}{\partial y}\left(t, \theta r \cos \varphi+\mu_{1}(t, \alpha), \theta r \sin \varphi+\mu_{2}(t, \alpha), \alpha\right) r \cos \varphi \sin \varphi+ \\
\left.\frac{\partial f_{2}}{\partial x}\left(t, \theta r \cos \varphi+\mu_{1}(t, \alpha), \theta r \sin \varphi+\mu_{2}(t, \alpha), \alpha\right) r \cos \varphi \sin \varphi\right) d \theta \\
\geq \int_{0}^{1}\left(\gamma r \cos ^{2} \varphi+\gamma r \sin ^{2} \varphi-\frac{\gamma}{2} r \cos \varphi \sin \varphi\right) d \theta \geq r \frac{\gamma}{2}
\end{gathered}
$$


Applying polar coordinates, we see that $\nu$ is past repulsive. The asserted relations concerning $\mathcal{R}_{\mu(\cdot, \alpha)}$ and the existence of the past attractors follow directly from Lemma 5.4 (ii).

As in the previous section, these lemmata lead to the existence of total past bifurcations and transitions.

Theorem 7.3. The following statements are fulfilled:

(i) If $a\left(\alpha_{1}\right)<0$, then there exists a continuous function $\mu: D \subset \mathbb{R} \times\left(\alpha_{0}, \alpha_{1}\right] \rightarrow$ $\mathbb{R}^{2}$ such that $\mu(\cdot, \alpha)$ is a past attractive solution of $(7.2)$. We have a total past bifurcation, since

$$
\lim _{\alpha \searrow \alpha_{0}} d\left(\mathcal{A}_{\mu(\cdot, \alpha)} \mid\{0\}\right)=0 .
$$

Furthermore, for all $\alpha \in\left(\alpha_{0}, \alpha_{1}\right]$, there exists a past repeller $R(\alpha)$. We also have a past repeller transition, since

$$
\lim _{\alpha \searrow \alpha_{0}} d\left(\limsup _{t \rightarrow-\infty} R(\alpha, t) \mid\left\{\left(x_{0}, y_{0}\right)\right\}\right)=0 .
$$

(ii) If $a\left(\alpha_{1}\right)>0$, then there exists a continuous function $\mu: D \subset \mathbb{R} \times\left(\alpha_{0}, \alpha_{1}\right] \rightarrow$ $\mathbb{R}^{2}$ such that $\mu(\cdot, \alpha)$ is a past repulsive solution of $(7.2)$. We have a total past bifurcation, since

$$
\lim _{\alpha \searrow \alpha_{0}} d\left(\mathcal{R}_{\mu(\cdot, \alpha)} \mid\{0\}\right)=0 .
$$

Furthermore, for all $\alpha \in\left(\alpha_{0}, \alpha_{1}\right]$, there exists a past attractor $A(\alpha)$. We also have a past attractor transition, since

$$
\lim _{\alpha \searrow \alpha_{0}} d\left(\limsup _{t \rightarrow-\infty} A(\alpha, t) \mid\left\{\left(x_{0}, y_{0}\right)\right\}\right)=0 .
$$

Proof. See proof of Theorem 6.3.

\section{Conclusion}

In this article, we have introduced new notions of attractivity and bifurcation for nonautonomous differential equations, and we have seen that these notions lead to bifurcation results for asymptotically autonomous equations in case the underlying autonomous system admits a bifurcation of saddle node, transcritical, pitchfork or Hopf type. These considerations are, together with the one-dimensional bifurcation patterns as discussed in [27], a first step towards a nonautonomous bifurcation theory. Having the basic tools available, further research in nonautonomous bifurcation theory will concentrate on the study of high-dimensional systems, e.g., by applying center manifold reduction methods, and the development of a nonautonomous normal form theory.

As outlined in the Introduction, there have been numerous other approaches to nonautonomous bifurcation theory in the last ten years, and we distinguish between topological skew product flows and approaches without imposing special hypotheses on the base set such as compactness.

In the bifurcation theory of nonautonomous dynamical systems where the base set is supposed to have a certain topological structure, one distinguishes between attractor-repeller bifurcations and bifurcations of solutions. An attractor-repeller bifurcation either occurs if a nontrivial attractor or repeller, respectively, shrinks 
down to a trivial object by variation of the parameter (this corresponds to the notion of transition), or if an attractor bifurcates from a repeller in the sense of Hausdorff distance. Note that the attractors and repellers under consideration are autonomous objects of the skew product flow. In [14, 9, 8], for one-dimensional nonautonomous differential equations with strictly ergodic time dependence (e.g., quasi-periodic equations are of this type), attractor-repeller bifurcations are considered. Bifurcations of attractors and repellers are also studied in [13, 12] for deterministic counterparts of the Two-Step-Bifurcation-Pattern. These considerations are based on the studies of Ludwig Arnold and his coworkers in the context of stochastic differential equations (see [2]). In [11], a bifurcation of nonchaotic strange attractors of a quasi-periodic differential equation is verified, both numerically and analytically. A bifurcation (of pitchfork and transcritical type) of almost periodic solutions of an almost periodic ordinary differential equation is examined in [15]. Furthermore, one-dimensional bifurcations of bounded solutions are considered in [22] in the context of strict ergodicity.

There have also been several approaches in the study of bifurcation phenomena of nonautonomous dynamical systems where no special hypotheses concerning the base set are made. In [16], a nonautonomous bifurcation is understood as a (continuous or discontinuous) transition from a nontrivial (global) pullback attractor to a trivial pullback attractor. In [17], for nonautonomous differential equations, notions of Lyapunov pullback-stable and Lyapunov pullback-unstable solutions are introduced, and bifurcations in form of merging processes of two distinct solutions with different stability behavior are studied by means of relatively simple examples. In their recent paper [19], the three authors found sufficient conditions for the Taylor coefficients of the right hand side of one-dimensional differential equations which guarantee the existence of such bifurcations. Moreover, in [23, 24], the bifurcation and continuation of bounded solutions of nonautonomous difference equations is studied using a functional analytic approach.

Acknowledgement. The author wishes to thank an anonymous referee for valuable suggestions leading to an improvement of this paper.

\section{REFERENCES}

[1] R. H. Abraham, J. E. Marsden, and T. Ratiu, Manifolds, Tensor Analysis, and Applications, Springer, New York, 1988.

[2] L. Arnold, Random Dynamical Systems, Springer, Berlin, Heidelberg, New York, 1998.

[3] J. P. Aubin and H. Frankowska, Set-Valued Analysis, Systems and Control: Foundations and Applications, vol. 2, Birkhäuser, Boston, 1990.

[4] B. Aulbach, M. Rasmussen, and S. Siegmund, Invariant manifolds as pullback attractors of nonautonomous differential equations, Discrete and Continuous Dynamical Systems 15 (2006), no. 2, 579-596.

[5] A. Berger and S. Siegmund, Uniformly attracting solutions of nonautonomous differential equations, to appear in: Nonlinear Analysis. Theory, Methods \& Applications.

[6] D. N. Cheban, P. E. Kloeden, and B. Schmalfuß, Pullback attractors in dissipative nonautonomous differential equations under discretization, Journal of Dynamics and Differential Equations 13 (2001), no. 1, 185-213.

[7] M. Dellnitz and A. Hohmann, A subdivision algorithm for the computation of unstable manifolds and global attractors, Numerische Mathematik 75 (1997), 293-317.

[8] R. Fabbri and R. A. Johnson, On a saddle-node bifurcation in a problem of quasi-periodic harmonic forcing, EQUADIFF 2003. Proceedings of the International Conference on Differential Equations, Hasselt, Belgium (F. Dumortier, H. Broer, J. Mawhin, A. Vanderbauwhede, and V. Lunel, eds.), 2005, pp. 839-847. 
[9] R. Fabbri, R. A. Johnson, and F. Mantellini, A nonautonomous saddle-node bifurcation pattern, Stochastics and Dynamics 4 (2004), no. 3, 335-350.

[10] F. Flandoli and B. Schmalfuß, Random attractors for the 3-D stochastic Navier-Stokes equation with mulitiplicative white noise, Stochastics and Stochastics Reports 59 (1996), no. 1-2, $21-45$.

[11] P. Glendinning, Non-smooth pitchfork bifurcations, Discrete and Continuous Dynamical Systems B 4 (2004), no. 2, 457-464.

[12] R. A. Johnson, An application of topological dynamics to bifurcation theory, Topological Dynamics and Applications. A volume in honor of Robert Ellis (M. G. Nerurkar, D. P. Dokken, and D. B. Ellis, eds.), Contemporary Mathematics, vol. 215, American Mathematical Society, Providence, Rhode Island, 1998, pp. 323-334.

[13] R. A. Johnson, P. E. Kloeden, and R. Pavani, Two-step transition in nonautonomous bifurcations: An explanation, Stochastics and Dynamics 2 (2002), no. 1, 67-92.

[14] R. A. Johnson and F. Mantellini, A nonautonomous transcritical bifurcation problem with an application to quasi-periodic bubbles, Discrete and Continuous Dynamical Systems 9 (2003), no. 1, 209-224.

[15] P. E. Kloeden, Pitchfork and transcritical bifurcations in systems with homogeneous nonlinearities and an almost periodic time coefficient, Communications on Pure and Applied Analysis 3 (2004), no. 2, 161-173.

[16] P. E. Kloeden and S. Siegmund, Bifurcations and continuous transitions of attractors in autonomous and nonautonomous systems, International Journal of Bifurcation and Chaos 15 (2005), no. 3, 743-762.

[17] J. A. Langa, J. C. Robinson, and A. Suárez, Stability, instability and bifurcation phenomena in non-autonomous differential equations, Nonlinearity 15 (2002), no. 3, 887-903.

[18] J. A. Langa, J. C. Robinson, and A. Suárez, Forwards and pullback behaviour of a nonautonomous Lotka-Volterra system, Nonlinearity 16 (2003), no. 4, 1277-1293.

[19] _ Bifurcations in non-autonomous scalar equations, Journal of Differential Equations 221 (2006), no. 1, 1-35.

[20] L. Markus, Asymptotically autonomous differential systems, Contributions to the Theory of Nonlinear Oscillations III, Annals of Mathematical Studies (S. Lefschetz, ed.), vol. 36, Princeton University Press, 1956, pp. 17-29.

[21] J. E. Marsden and M. McCracken, The Hopf Bifurcation and its Applications, Applied Mathematical Sciences, vol. 19, Springer, Berlin, Heidelberg, New York, 1976.

[22] C. Núñez and R. Obaya, A non-autonomous bifurcation theory for deterministic scalar differential equations, to appear in: Discrete and Continuous Dynamical Systems A.

[23] C. Pötzsche, Nonautonomous continuation and bifurcation of bounded solutions I: Difference equations, Manuscript.

[24] _ Robustness of hyperbolic solutions under parametric perturbations, to appear in: Journal of Difference Equations and Applications.

[25] M. Rasmussen, Towards a bifurcation theory for nonautonomous difference equations, Journal of Difference Equations and Applications 12 (2006), no. 3-4, 297-312.

[26] __ Attractivity and Bifurcation for Nonautonomous Dynamical Systems, Springer Lecture Notes in Mathematics, vol. 1907, Springer, Berlin, Heidelberg, New York, 2007.

[27] _ Nonautonomous bifurcation patterns for one-dimensional differential equations, Journal of Differential Equations 234 (2007), no. 1, 267-288.

[28] R. Temam, Infinite-Dimensional Dynamical Systems in Mechanics and Physics, Applied Mathematical Sciences, vol. 68, Springer, New York, 1988.

[29] H. Thieme, Asymptotically autonomous differential equations in the plane, Rocky Mountain Journal of Mathematics 24 (1994), no. 1, 351-380.

Martin Rasmussen, Department of Mathematics, University of Augsburg, D-86135 Augsburg, Germany

E-mail address: martin.rasmussen@math.uni-augsburg.de 